\title{
Zone-Boundary Phonon in Graphene and Nanotube
}

\author{
Hidekatsu SuzuURA and Tsuneya ANDO ${ }^{1}$ \\ Division of Applied Physics, Graduate School of Engineering \\ Hokkaido University, Sapporo 060-8628 \\ ${ }^{1}$ Department of Physics, Tokyo Institute of Technology \\ 2-12-1 Ookayama, Meguro-ku, Tokyo 152-8551
}

\begin{abstract}
Zone-boundary phonons are described in a valence-force-field model and then converted into a continuum model. Only a mode with the highest frequency corresponding to a Kekulé-type distortion gives rise to inter-valley electron scattering between $K$ and $K$ ' points. An effective Hamiltonian for this interaction is obtained and used for the estimation of inter-valley scattering in graphene and nanotubes.
\end{abstract}

Keywords: carbon nanotube, graphene, two-dimensional graphite, electron-phonon interaction, intervalley scattering, optical phonon

\section{$\S 1$. Introduction}

The electron-phonon interaction plays important roles in various phenomena in graphene and carbon nanotubes. In a previous work, a continuum model of longwavelength acoustic phonons and an effective Hamiltonian for electron-phonon interactions were constructed. ${ }^{1)}$ A similar continuum model was developed for longwavelength optical phonons near the Brillouin-zone center. $^{2,3)}$ The purpose of this paper is to develop a continuum model for zone-boundary phonons appropriate for studying effects of inter-valley electron scattering within an effective-mass or a $\boldsymbol{k} \cdot \boldsymbol{p}$ scheme.

In an effective-mass approximation, an electron in a graphite monolayer is described by Weyl's equation for a massless neutrino. ${ }^{4,5)}$ Transport properties in such an exotic electronic structure are quite intriguing, and the conductivity with/without a magnetic field including the Hall effect, ${ }^{6,7)}$ the dynamical transport, ${ }^{8)}$ and quantum correction to the conductivity $\left.{ }^{9}\right)$ were investigated theoretically. The results show that the system exhibits various characteristic behaviors different from conventional two-dimensional systems. ${ }^{10)}$ Quite recently, this single layer graphite was fabricated, ${ }^{11)}$ and the magnetotransport was measured including the integer quantum Hall effect, demonstrating the validity of the neutrino description of the electronic states. ${ }^{12,13)}$ The graphene has become the subject of extensive experimental ${ }^{14-24)}$ and theoretical investigations. ${ }^{3,25-41)}$

Carbon nanotubes, which are rolled graphene sheets, also exhibit interesting transport properties. In metallic nanotubes, in particular, the backward scattering is entirely suppressed for scatterers with potential range larger than the lattice constant and the conductance is quantized into $2 e^{2} / \pi \hbar .^{42,43)}$ This intriguing fact was related to Berry's phase acquired by a rotation in the wave-vector space. ${ }^{44)}$ When several bands are occupied, a perfectly conducting channel transmitting through the system without being scattered back is present. ${ }^{45)}$ Roles of special time-reversal symmetry and crossover due to symmetry breaking perturbations were discussed. ${ }^{46-50)}$ Metallic nanotubes are almost ballistic with a mean free path exceeding $1 \mu \mathrm{m}$ even at room temperature. ${ }^{1)}$

Experiments on the high-field transport in singlewall nanotubes were performed, demonstrating the importance of the scattering by optical phonons in limiting the ballistic behavior in strong electric fields. ${ }^{51-53)}$ There have been various theoretical calculations on interaction with optical phonons using tight-binding models ${ }^{51-58)}$ and LDA band-calculations. ${ }^{59)}$ However, these works have not provided a scheme suitable for calculations in the effective-mass formulation, giving direct physical insight into roles of zone-boundary phonons causing intervalley scattering.

In this paper, we shall start with a valence-forcefield model for phonons in graphene, derive a continuum model of zone-boundary phonons, and obtain an effective-mass Hamiltonian describing intervalley electron scattering. The paper is organized as follows: In $\S 2$ a very brief review is given on the derivation of an effective-mass equation from a nearest-neighbor tight-binding model and on a valence-force-field model. Phonon modes at zone boundaries are obtained and then an effective interaction Hamiltonian is derived. It is shown that only the mode with highest frequency contributes to the electron-phonon interaction. The relaxation time due to intervalley scattering by the zone-boundary phonon is estimated and compared with that due to zone-center phonons in graphene in $\S 3$ and in carbon nanotubes in $\S 4$. Effects of interaction on frequency and broadening of zone-boundary phonon are briefly discussed in $\S 5$. A short summary is given in $\S 6$.

\section{$\S 2$. Formulation}

\subsection{Effective-Mass Approximation}

The structure of 2D graphite sheet or graphene is shown in Fig. 1 (a). A unit cell contains two carbon atoms, which are denoted by $\mathrm{A}$ and $\mathrm{B}$. In the coordinates system $x^{\prime} y^{\prime}$ fixed onto the graphene, the primitive translation vectors are $\boldsymbol{a}=(1,0) a$ and $\boldsymbol{b}=(-1 / 2, \sqrt{3} / 2) a$ with lattice constant $a=0.246 \mathrm{~nm}$. Three vectors connecting neighboring $\mathrm{A}$ and $\mathrm{B}$ atoms with bond length $b=a / \sqrt{3}$ shown in Fig. 1 (a) are given by $\boldsymbol{\tau}_{1}=(0,1 / \sqrt{3}) a, \boldsymbol{\tau}_{2}=$ 
$(-1 / 2,-1 / 2 \sqrt{3}) a$, and $\tau_{3}=(1 / 2,-1 / 2 \sqrt{3}) a$. The first Brillouin zone is shown in Fig. 1 (b). The wave vectors of the $\mathrm{K}$ and $\mathrm{K}^{\prime}$ points are given by $\boldsymbol{K}=(2 \pi / a)(1 / 3,1 / \sqrt{3})$ and $\boldsymbol{K}^{\prime}=(2 \pi / a)(2 / 3,0)$, respectively.

Although an effective-mass approximation or $\boldsymbol{k} \cdot \boldsymbol{p}$ scheme is more general, we shall start with a nearestneighbor tight-binding model to describe the $\pi$ band where the Fermi level lies. Let $\psi_{A}\left(\boldsymbol{R}_{A}\right)$ be the amplitude of the $\pi$ orbital of an $\mathrm{A}$ atom at $\boldsymbol{R}_{A}=n_{a} \boldsymbol{a}+n_{b} \boldsymbol{b}+\boldsymbol{\tau}_{1}$ and $\psi_{B}\left(\boldsymbol{R}_{B}\right)$ be that of a $\mathrm{B}$ atom at $\boldsymbol{R}_{B}=n_{a} \boldsymbol{a}+n_{b} \boldsymbol{b}$. Then, the equation of motion is given by

$$
\begin{aligned}
& \varepsilon \psi_{A}\left(\boldsymbol{R}_{A}\right)=-\gamma_{0} \sum_{l=1}^{3} \psi_{B}\left(\boldsymbol{R}_{A}-\boldsymbol{\tau}_{l}\right), \\
& \varepsilon \psi_{B}\left(\boldsymbol{R}_{B}\right)=-\gamma_{0} \sum_{l=1}^{3} \psi_{A}\left(\boldsymbol{R}_{B}+\boldsymbol{\tau}_{l}\right) .
\end{aligned}
$$

For states in the vicinity of the Fermi level $\varepsilon=0$, the wavefunction is written as ${ }^{5)}$

$$
\begin{aligned}
& \psi_{A}\left(\boldsymbol{R}_{A}\right)=\mathrm{e}^{\mathrm{i} \boldsymbol{K} \cdot \boldsymbol{R}_{A}} F_{A}^{K}\left(\boldsymbol{R}_{A}\right)+\mathrm{e}^{\mathrm{i} \eta} \mathrm{e}^{\mathrm{i} \boldsymbol{K}^{\prime} \cdot \boldsymbol{R}_{A}} F_{A}^{K^{\prime}}\left(\boldsymbol{R}_{A}\right), \\
& \psi_{B}\left(\boldsymbol{R}_{B}\right)=-\omega \mathrm{e}^{\mathrm{i} \eta} \mathrm{e}^{\mathrm{i} \boldsymbol{K} \cdot \boldsymbol{R}_{B}} F_{B}^{K}\left(\boldsymbol{R}_{B}\right)+\mathrm{e}^{\mathrm{i} \boldsymbol{K}^{\prime} \cdot \boldsymbol{R}_{B}} F_{B}^{K^{\prime}}\left(\boldsymbol{R}_{B}\right),
\end{aligned}
$$

in terms of the slowly-varying envelope functions $F_{A}^{K}$, $F_{B}^{K}, F_{A}^{K^{\prime}}$, and $F_{B}^{K^{\prime}}$, where $\eta$ is a chiral angle as shown in Fig. 1 (a) and $\omega=\exp (2 \pi i / 3)$. The envelope functions satisfy the Schrödinger equation

$$
\left(\begin{array}{cc}
\gamma(\boldsymbol{\sigma} \cdot \hat{\boldsymbol{k}}) & 0 \\
0 & \gamma\left(\boldsymbol{\sigma}^{*} \cdot \hat{\boldsymbol{k}}\right)
\end{array}\right)\left(\begin{array}{c}
\boldsymbol{F}^{K}(\boldsymbol{r}) \\
\boldsymbol{F}^{K^{\prime}}(\boldsymbol{r})
\end{array}\right)=\varepsilon\left(\begin{array}{c}
\boldsymbol{F}^{K}(\boldsymbol{r}) \\
\boldsymbol{F}^{K^{\prime}}(\boldsymbol{r})
\end{array}\right),
$$

where $\hat{\boldsymbol{k}}=-\mathrm{i} \boldsymbol{\nabla}, \boldsymbol{\sigma}=\left(\sigma_{x}, \sigma_{y}\right)$ with $\sigma_{x}$ and $\sigma_{y}$ being Pauli spin matrices, and

$$
\boldsymbol{F}^{K}=\left(\begin{array}{c}
F_{A}^{K} \\
F_{B}^{K}
\end{array}\right), \quad \boldsymbol{F}^{K^{\prime}}=\left(\begin{array}{c}
F_{A}^{K^{\prime}} \\
F_{B}^{K^{\prime}}
\end{array}\right) .
$$

A brief review on the derivation is given in Appendix A. Note that eq. (2.4) itself is quite general and not limited to the nearest-neighbor tight-binding model.

\subsection{Valence-Force-Field Model}

In a valence-force-field model, two kinds of interatomic forces are considered. ${ }^{1,54,60)}$ The first is the force associated with the change in the bond length. The corresponding potential energy becomes

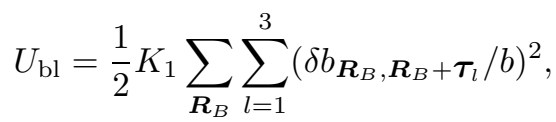

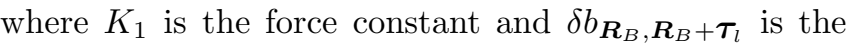
bond-length change for the bond between atoms at $\boldsymbol{R}_{B}$ and $\boldsymbol{R}_{B}+\boldsymbol{\tau}_{l}$. The phonon spectrum obtained by including $K_{1}$ was reported previously. ${ }^{61,35)}$ The second is the force associated with the change in the bond angle. Let $\delta \theta_{l m}\left(\boldsymbol{R}_{B}\right)$ be the deviation of the bond angle determined by three neighboring atoms $\boldsymbol{R}_{B}, \boldsymbol{R}_{B}+\boldsymbol{\tau}_{l}$, and $\boldsymbol{R}_{B}+\boldsymbol{\tau}_{m}$ from $2 \pi / 3$. Then, the potential energy becomes

$$
U_{\mathrm{ba}}=\frac{1}{2} K_{2} \sum_{\boldsymbol{R}_{A}} \sum_{(l, m)} \delta \theta_{l m}\left(\boldsymbol{R}_{A}\right)^{2}+\frac{1}{2} K_{2} \sum_{\boldsymbol{R}_{B}} \sum_{(l, m)} \delta \theta_{l m}\left(\boldsymbol{R}_{B}\right)^{2},
$$

where $K_{2}$ is the force constant and the summation is over $(l, m)=(1,2),(2,3)$, and $(3,1)$, i.e., over three angles around each atom.

Let us define

$$
\begin{aligned}
& \boldsymbol{u}_{A}\left(\boldsymbol{R}_{A}\right)=\boldsymbol{u}_{A}(\boldsymbol{q}) \mathrm{e}^{\mathrm{i} \boldsymbol{q} \cdot \boldsymbol{R}_{A}}, \\
& \boldsymbol{u}_{B}\left(\boldsymbol{R}_{B}\right)=\boldsymbol{u}_{B}(\boldsymbol{q}) \mathrm{e}^{\mathrm{i} \boldsymbol{q} \cdot \boldsymbol{R}_{B}},
\end{aligned}
$$

and the four-component vector

$$
\boldsymbol{U}(\boldsymbol{q})=\left(\begin{array}{c}
u_{A x}(\boldsymbol{q}) \\
u_{A y}(\boldsymbol{q}) \\
u_{B x}(\boldsymbol{q}) \\
u_{B y}(\boldsymbol{q})
\end{array}\right)
$$

Then, the phonon equation of motion is written as

$$
M \omega^{2}(\boldsymbol{q}) \boldsymbol{U}(\boldsymbol{q})=\mathcal{H}_{\mathrm{ph}}(\boldsymbol{q}) \boldsymbol{U}(\boldsymbol{q}),
$$

where $M$ is the mass of a carbon atom $\left(M=1.993 \times 10^{-23}\right.$ g) and $\mathcal{H}_{\mathrm{ph}}$ is the effective phonon Hamiltonian.

The phonon Hamiltonian is written as

$$
\mathcal{H}_{\mathrm{ph}}(\boldsymbol{q})=\mathcal{H}_{\mathrm{bl}}(\boldsymbol{q})+\mathcal{H}_{\mathrm{ba}}(\boldsymbol{q}),
$$

with

$$
\mathcal{H}_{\mathrm{bl}}(\boldsymbol{q})=K_{1}\left(\begin{array}{cccc}
3 / 2 & 0 & h_{1}(\boldsymbol{q}) & h_{2}(\boldsymbol{q}) \\
0 & 3 / 2 & h_{2}(\boldsymbol{q}) & h_{3}(\boldsymbol{q}) \\
h_{1}(\boldsymbol{q})^{*} & h_{2}(\boldsymbol{q})^{*} & 3 / 2 & 0 \\
h_{2}(\boldsymbol{q})^{*} & h_{3}(\boldsymbol{q})^{*} & 0 & 3 / 2
\end{array}\right),
$$

and

$$
\mathcal{H}_{\mathrm{ba}}(\boldsymbol{q})=K_{2}\left(\begin{array}{cccc}
h_{4}(\boldsymbol{q}) & h_{5}(\boldsymbol{q})^{*} & 6 h_{3}(\boldsymbol{q}) & -6 h_{2}(\boldsymbol{q}) \\
h_{5}(\boldsymbol{q}) & h_{6}(\boldsymbol{q}) & -6 h_{2}(\boldsymbol{q}) & 6 h_{1}(\boldsymbol{q}) \\
6 h_{3}(\boldsymbol{q})^{*} & -6 h_{2}(\boldsymbol{q})^{*} & h_{4}(\boldsymbol{q}) & h_{5}(\boldsymbol{q}) \\
-6 h_{2}(\boldsymbol{q})^{*} & 6 h_{1}(\boldsymbol{q})^{*} & h_{5}(\boldsymbol{q})^{*} & h_{6}(\boldsymbol{q})
\end{array}\right),
$$

where

$$
\begin{aligned}
& h_{1}=-\frac{3}{2} \exp \left(\mathrm{i} \frac{q_{y} a}{2 \sqrt{3}}\right) \cos \left(\frac{q_{x} a}{2}\right) \\
& h_{2}=-\mathrm{i} \frac{\sqrt{3}}{2} \exp \left(\mathrm{i} \frac{q_{y} a}{2 \sqrt{3}}\right) \sin \left(\frac{q_{x} a}{2}\right) \\
& h_{3}=-\exp \left(-\mathrm{i} \frac{q_{y} a}{\sqrt{3}}\right)-\frac{1}{2} \exp \left(\mathrm{i} \frac{q_{y} a}{2 \sqrt{3}}\right) \cos \left(\frac{q_{x} a}{2}\right) \\
& h_{4}=7+\sin ^{2}\left(\frac{q_{x} a}{2}\right)+2 \cos \left(\frac{q_{x} a}{2}\right) \cos \left(\frac{\sqrt{3} q_{y} a}{2}\right) \\
& h_{5}=-\mathrm{i} \sqrt{3} \exp \left(\mathrm{i} \frac{\sqrt{3} q_{y} a}{2}\right) \sin \left(\frac{q_{x} a}{2}\right)+\mathrm{i} \frac{\sqrt{3}}{2} \sin q_{x} a \\
& h_{6}=9-3 \sin ^{2}\left(\frac{q_{x} a}{2}\right) .
\end{aligned}
$$

\subsection{Phonons at K' Point}

At a $K^{\prime}$ point $\boldsymbol{q}=\boldsymbol{K}^{\prime}$, we have

$$
\begin{aligned}
\mathcal{H}_{\mathrm{ph}}^{K^{\prime}} & =\frac{3}{4} K_{1}\left(\begin{array}{cc}
2 & \sigma_{z}-\mathrm{i} \sigma_{x} \\
\sigma_{z}+\mathrm{i} \sigma_{x} & 2
\end{array}\right) \\
& +\frac{9}{4} K_{2}\left(\begin{array}{cc}
3-\sigma_{y} & -2\left(\sigma_{z}-\mathrm{i} \sigma_{x}\right) \\
-2\left(\sigma_{z}+\mathrm{i} \sigma_{x}\right) & 3+\sigma_{y}
\end{array}\right) .
\end{aligned}
$$

The corresponding eigenfrequencies are

$$
\omega_{1}^{2}=3 \frac{K_{1}}{M}, \quad \omega_{2}^{2}=\frac{3}{2} \frac{K_{1}}{M}+\frac{9}{2} \frac{K_{2}}{M}, \quad \omega_{3}^{2}=18 \frac{K_{2}}{M},
$$

in the order of decreasing frequency. The corresponding 
eigenvectors are

$$
\begin{gathered}
\boldsymbol{e}_{1}^{K^{\prime}}=\frac{1}{2}\left(\begin{array}{c}
1 \\
-\mathrm{i} \\
1 \\
\mathrm{i}
\end{array}\right), \quad \boldsymbol{e}_{2 a}^{K^{\prime}}=\frac{1}{\sqrt{2}}\left(\begin{array}{c}
1 \\
\mathrm{i} \\
0 \\
0
\end{array}\right), \\
\boldsymbol{e}_{2 b}^{K^{\prime}}=\frac{1}{\sqrt{2}}\left(\begin{array}{c}
0 \\
0 \\
1 \\
-\mathrm{i}
\end{array}\right), \quad \boldsymbol{e}_{3}^{K^{\prime}}=\frac{1}{2}\left(\begin{array}{c}
1 \\
-\mathrm{i} \\
-1 \\
-\mathrm{i}
\end{array}\right) .
\end{gathered}
$$

The modes $g=2 a$ and $2 b$ are doubly degenerate. Therefore, we have to consider the correction of the Hamiltonian $\mathcal{H}_{\mathrm{ph}}^{(1)}$ to the lowest order in the wave vector relative to the $\mathrm{K}^{\prime}$ point. Taking the matrix elements of $\mathcal{H}_{\mathrm{ph}}^{(1)}$ for modes $g=2 a$ and $2 b$, we have the effective Hamiltonian

$$
\mathcal{H}_{\mathrm{ph}}=\frac{\sqrt{3}}{4}\left(K_{1}-6 K_{2}\right) a(\boldsymbol{\sigma} \cdot \boldsymbol{q}) .
$$

This shows that the modes $g=2$ have a linear dispersion crossing at the K' point, analogous to the electron dispersion. The modes $g=1$ and 3 have a quadratic dispersion at the $\mathrm{K}^{\prime}$ point and therefore their $q$ dependence can be safely neglected. Figure 2 shows a schematic illustration of phonon frequencies at the $K^{\prime}$ point.

The Hamiltonian for $\boldsymbol{q}=-\boldsymbol{K}^{\prime}$ is calculated as

$$
\begin{aligned}
\mathcal{H}_{\mathrm{ph}}^{-K^{\prime}} & =\frac{3}{4} K_{1}\left(\begin{array}{cc}
2 & \sigma_{z}+\mathrm{i} \sigma_{x} \\
\sigma_{z}-\mathrm{i} \sigma_{x} & 2
\end{array}\right) \\
& +\frac{9}{4} K_{2}\left(\begin{array}{cc}
3+\sigma_{y} & -2\left(\sigma_{z}+\mathrm{i} \sigma_{x}\right) \\
-2\left(\sigma_{z}-\mathrm{i} \sigma_{x}\right) & 3-\sigma_{y}
\end{array}\right) .
\end{aligned}
$$

This is related to that for $\boldsymbol{q}=\boldsymbol{K}^{\prime}$ through

$$
\mathcal{H}_{\mathrm{ph}}^{-K^{\prime}}=\mathcal{H}_{\mathrm{ph}}^{K^{\prime} *}
$$

and the eigenvectors are

$$
e_{g}^{-K^{\prime}}=e_{g}^{K^{\prime} *}
$$

for $g=1,2 a, 2 b$, and 3 .

\subsection{Phonons at $K$ point}

The Hamiltonian at a $\mathrm{K}$ point $\boldsymbol{q}=\boldsymbol{K}$ is calculated as

$$
\mathcal{H}_{\mathrm{ph}}^{K}=\left(\begin{array}{cc}
\omega^{-1} & 0 \\
0 & 1
\end{array}\right) \mathcal{H}_{\mathrm{ph}}^{-K^{\prime}}\left(\begin{array}{ll}
\omega & 0 \\
0 & 1
\end{array}\right),
$$

and the eigen vectors are

$$
\boldsymbol{e}_{g}^{K}=\left(\begin{array}{cc}
\omega^{-1} & 0 \\
0 & 1
\end{array}\right) \boldsymbol{e}_{g}^{-K^{\prime}}
$$

Because the point $\boldsymbol{K}$ is related to the point $-\boldsymbol{K}^{\prime}$ through the reciprocal lattice vector $\boldsymbol{K}+\boldsymbol{K}^{\prime}$, the displacements $\boldsymbol{u}_{A}$ and $\boldsymbol{u}_{B}$ should be the same within a common phase factor, i.e.,

$$
\begin{aligned}
& \boldsymbol{u}_{A}(\boldsymbol{K}) \exp \left(\mathrm{i} \boldsymbol{K} \cdot \boldsymbol{R}_{A}\right)=\mathrm{e}^{\mathrm{i} \varphi} \boldsymbol{u}_{A}\left(-\boldsymbol{K}^{\prime}\right) \exp \left(-\mathrm{i} \boldsymbol{K}^{\prime} \cdot \boldsymbol{R}_{A}\right) \\
& \boldsymbol{u}_{B}(\boldsymbol{K}) \exp \left(\mathrm{i} \boldsymbol{K} \cdot \boldsymbol{R}_{B}\right)=\mathrm{e}^{\mathrm{i} \varphi} \boldsymbol{u}_{B}\left(-\boldsymbol{K}^{\prime}\right) \exp \left(-\mathrm{i} \boldsymbol{K}^{\prime} \cdot \boldsymbol{R}_{B}\right)
\end{aligned}
$$

For the present choice of the coordinates system, this gives

$$
\left(\begin{array}{l}
\boldsymbol{u}_{A}(\boldsymbol{K}) \\
\boldsymbol{u}_{B}(\boldsymbol{K})
\end{array}\right)=\mathrm{e}^{\mathrm{i} \varphi}\left(\begin{array}{cc}
\omega^{-1} & 0 \\
0 & 1
\end{array}\right)\left(\begin{array}{l}
\boldsymbol{u}_{A}\left(-\boldsymbol{K}^{\prime}\right) \\
\boldsymbol{u}_{B}\left(-\boldsymbol{K}^{\prime}\right)
\end{array}\right)
$$

The above relations (2.27) and (2.28) are certainly consistent with this requirement.

\subsection{Zone-Boundary Phonon}

In general, the lattice displacements are written as

$$
\begin{aligned}
&\left(\begin{array}{c}
\boldsymbol{u}_{A}\left(\boldsymbol{R}_{A}\right) \\
\boldsymbol{u}_{B}\left(\boldsymbol{R}_{B}\right)
\end{array}\right)=\sum_{g, \boldsymbol{q}} \sqrt{\frac{\hbar}{2 N M \omega_{g}(\boldsymbol{q})}}\left(b_{g, \boldsymbol{q}}+b_{g,-\boldsymbol{q}}^{\dagger}\right) \\
& \times\left(\begin{array}{c}
\boldsymbol{e}_{A g}(\boldsymbol{q}) \mathrm{e}^{\mathrm{i} \boldsymbol{q} \cdot \boldsymbol{R}_{A}} \\
\boldsymbol{e}_{B g}(\boldsymbol{q}) \mathrm{e}^{\mathrm{i} \boldsymbol{q} \cdot \boldsymbol{R}_{B}}
\end{array}\right),
\end{aligned}
$$

where $N$ is the total number of unit cells in the system and $b_{g, \boldsymbol{q}}$ and $b_{g, \boldsymbol{q}}^{\dagger}$ are the destruction and creation operators of a phonon with mode $g$ and wave vector $\boldsymbol{q}$. This can be separated into the contribution of $\boldsymbol{q} \sim \boldsymbol{K}^{\prime}$ and $\boldsymbol{q} \sim-\boldsymbol{K}^{\prime}$ as

$$
\begin{aligned}
\boldsymbol{u}_{A}\left(\boldsymbol{R}_{A}\right) & =\boldsymbol{u}_{A}^{K^{\prime}}\left(\boldsymbol{R}_{A}\right) \mathrm{e}^{\mathrm{i} \boldsymbol{K}^{\prime} \cdot \boldsymbol{R}_{A}}+\boldsymbol{u}_{A}^{-K^{\prime}}\left(\boldsymbol{R}_{A}\right) \mathrm{e}^{-\mathrm{i} \boldsymbol{K}^{\prime} \cdot \boldsymbol{R}_{A}}, \\
\boldsymbol{u}_{B}\left(\boldsymbol{R}_{B}\right) & =\boldsymbol{u}_{B}^{K^{\prime}}\left(\boldsymbol{R}_{B}\right) \mathrm{e}^{\mathrm{i} \boldsymbol{K}^{\prime} \cdot \boldsymbol{R}_{B}}+\boldsymbol{u}_{B}^{-K^{\prime}}\left(\boldsymbol{R}_{B}\right) \mathrm{e}^{-\mathrm{i} \boldsymbol{K}^{\prime} \cdot \boldsymbol{R}_{B}},
\end{aligned}
$$

where $\boldsymbol{u}_{A}^{K^{\prime}}(\boldsymbol{r})$, etc. are the slowly-varying part of the displacement given by

$$
\begin{aligned}
& \left(\begin{array}{c}
\boldsymbol{u}_{A}^{K^{\prime}}(\boldsymbol{r}) \\
\boldsymbol{u}_{B}^{K^{\prime}}(\boldsymbol{r})
\end{array}\right)=\sum_{g, \boldsymbol{q}} \sqrt{\frac{\hbar}{2 N M \omega_{g}(\boldsymbol{q})}}\left(\begin{array}{c}
\boldsymbol{e}_{A g}^{K^{\prime}}(\boldsymbol{q}) \\
\boldsymbol{e}_{B g}^{K^{\prime}}(\boldsymbol{q})
\end{array}\right) \\
& \times\left(b_{g, \boldsymbol{K}^{\prime}+\boldsymbol{q}}+b_{g,-\boldsymbol{K}^{\prime}-\boldsymbol{q}}^{\dagger}\right) \mathrm{e}^{\mathrm{i} \boldsymbol{q} \cdot \boldsymbol{r}}, \\
& \left(\begin{array}{c}
\boldsymbol{u}_{A}^{-K^{\prime}}(\boldsymbol{r}) \\
\boldsymbol{u}_{B}^{-K^{\prime}}(\boldsymbol{r})
\end{array}\right)=\sum_{g, \boldsymbol{q}} \sqrt{\frac{\hbar}{2 N M \omega_{g}(\boldsymbol{q})}}\left(\begin{array}{c}
\boldsymbol{e}_{A g}^{-K^{\prime}}(\boldsymbol{q}) \\
\boldsymbol{e}_{B g}^{-K^{\prime}}(\boldsymbol{q})
\end{array}\right) \\
& \times\left(b_{g,-\boldsymbol{K}^{\prime}+\boldsymbol{q}}+b_{g, \boldsymbol{K}^{\prime}-\boldsymbol{q}}^{\dagger}\right) \mathrm{e}^{\mathrm{i} \boldsymbol{q} \cdot \boldsymbol{r}},
\end{aligned}
$$

where $\boldsymbol{q}$ is limited to values satisfying $|\boldsymbol{q}| \ll(2 \pi / a)$ and $\omega_{g}(\boldsymbol{q})=\omega_{g}\left(-\boldsymbol{K}^{\prime}-\boldsymbol{q}\right)=\omega_{g}\left(\boldsymbol{K}^{\prime}+\boldsymbol{q}\right)$.

The lattice displacements can also be written as

$$
\begin{aligned}
& \boldsymbol{u}_{A}\left(\boldsymbol{R}_{A}\right)=\boldsymbol{u}_{A}^{K}\left(\boldsymbol{R}_{A}\right) \mathrm{e}^{\mathrm{i} \boldsymbol{K} \cdot \boldsymbol{R}_{A}}+\boldsymbol{u}_{A}^{K^{\prime}}\left(\boldsymbol{R}_{A}\right) \mathrm{e}^{\mathrm{i} \boldsymbol{K}^{\prime} \cdot \boldsymbol{R}_{A}}, \\
& \boldsymbol{u}_{B}\left(\boldsymbol{R}_{B}\right)=\boldsymbol{u}_{B}^{K}\left(\boldsymbol{R}_{B}\right) \mathrm{e}^{\mathrm{i} \boldsymbol{K} \cdot \boldsymbol{R}_{B}}+\boldsymbol{u}_{B}^{K^{\prime}}\left(\boldsymbol{R}_{B}\right) \mathrm{e}^{\mathrm{i} \boldsymbol{K}^{\prime} \cdot \boldsymbol{R}_{B}} .
\end{aligned}
$$

Let us define

$$
\begin{aligned}
& b_{K, g, \boldsymbol{q}}=b_{g,-\boldsymbol{K}^{\prime}+\boldsymbol{q}}, \quad b_{K, g, \boldsymbol{q}}^{\dagger}=b_{g,-\boldsymbol{K}^{\prime}+\boldsymbol{q}}^{\dagger}, \\
& b_{K^{\prime}, g, \boldsymbol{q}}=b_{g, \boldsymbol{K}^{\prime}+\boldsymbol{q}}, \quad b_{K^{\prime}, g, \boldsymbol{q}}^{\dagger}=b_{g, \boldsymbol{K}^{\prime}+\boldsymbol{q}}^{\dagger}
\end{aligned}
$$

Then, we have

$$
\begin{aligned}
\left(\begin{array}{c}
\boldsymbol{u}_{A}^{K}(\boldsymbol{r}) \\
\boldsymbol{u}_{B}^{K}(\boldsymbol{r})
\end{array}\right)=\sum_{g, \boldsymbol{q}} \sqrt{\frac{\hbar}{2 N M \omega_{g}(\boldsymbol{q})}}\left(\begin{array}{c}
\boldsymbol{e}_{A g}^{K}(\boldsymbol{q}) \\
\boldsymbol{e}_{B g}^{K}(\boldsymbol{q})
\end{array}\right) \\
\times\left(b_{K, g, \boldsymbol{q}}+b_{K^{\prime}, g,-\boldsymbol{q}}^{\dagger}\right) \mathrm{e}^{\mathrm{i} \boldsymbol{q} \cdot \boldsymbol{r}}, \\
\left(\begin{array}{c}
\boldsymbol{u}_{A}^{K^{\prime}}(\boldsymbol{r}) \\
\boldsymbol{u}_{B}^{K^{\prime}}(\boldsymbol{r})
\end{array}\right)=\sum_{g, \boldsymbol{q}} \sqrt{\frac{\hbar}{2 N M \omega_{g}(\boldsymbol{q})}}\left(\begin{array}{c}
\boldsymbol{e}_{A g}^{K^{\prime}}(\boldsymbol{q}) \\
\boldsymbol{e}_{B g}^{K^{\prime}}(\boldsymbol{q})
\end{array}\right) \\
\times\left(b_{K^{\prime}, g, \boldsymbol{q}}+b_{K, g,-\boldsymbol{q}}^{\dagger}\right) \mathrm{e}^{\mathrm{i} \boldsymbol{q} \cdot \boldsymbol{r}} .
\end{aligned}
$$

\subsection{Kekulé Distortion}

The mode $g=1$ with the highest frequency corresponds to a Kekulé-type distortion. In order to see 
this, we consider the corresponding lattice distortion explicitly. Let us first define

$$
f=\frac{1}{2}\left(\begin{array}{l}
1 \\
\mathrm{i}
\end{array}\right) \text {. }
$$

Then, we have

$$
\boldsymbol{e}_{1}^{K}=\left(\begin{array}{c}
\omega^{-1} \boldsymbol{f} \\
\boldsymbol{f}^{*}
\end{array}\right), \quad \boldsymbol{e}_{1}^{K^{\prime}}=\left(\begin{array}{c}
\boldsymbol{f}^{*} \\
\boldsymbol{f}
\end{array}\right) .
$$

Then, in terms of an appropriate complex number $\alpha=$ $|\alpha| \mathrm{e}^{\mathrm{i} \varphi}$, we have

$$
\begin{aligned}
& \boldsymbol{u}_{A}\left(\boldsymbol{R}_{A}\right)=\omega^{-1} \alpha^{*} \boldsymbol{f} \mathrm{e}^{\mathrm{i} \boldsymbol{K} \cdot \boldsymbol{R}_{A}}+\alpha \boldsymbol{f}^{*} \mathrm{e}^{\mathrm{i} \boldsymbol{K}^{\prime} \cdot \boldsymbol{R}_{A}}, \\
& \boldsymbol{u}_{B}\left(\boldsymbol{R}_{B}\right)=\alpha^{*} \boldsymbol{f}^{*} \mathrm{e}^{\mathrm{i} \boldsymbol{K} \cdot \boldsymbol{R}_{B}}+\alpha \boldsymbol{f} \mathrm{e}^{\mathrm{i} \boldsymbol{K}^{\prime} \cdot \boldsymbol{R}_{B}} .
\end{aligned}
$$

Consider the sites $\boldsymbol{R}_{B}=0$ and $\boldsymbol{R}_{A}=\boldsymbol{\tau}_{1}, \boldsymbol{\tau}_{2}$, and $\boldsymbol{\tau}_{3}$. Then, we have

$$
\begin{aligned}
& \boldsymbol{u}_{A}\left(\boldsymbol{\tau}_{1}\right)-\boldsymbol{u}_{B}(0)=2|\alpha| \sin \varphi \frac{\sqrt{3}}{a} \boldsymbol{\tau}_{1}, \\
& \boldsymbol{u}_{A}\left(\boldsymbol{\tau}_{2}\right)-\boldsymbol{u}_{B}(0)=2|\alpha| \sin \left(\varphi+\frac{2 \pi}{3}\right) \frac{\sqrt{3}}{a} \boldsymbol{\tau}_{2}, \\
& \boldsymbol{u}_{A}\left(\boldsymbol{\tau}_{3}\right)-\boldsymbol{u}_{B}(0)=2|\alpha| \sin \left(\varphi-\frac{2 \pi}{3}\right) \frac{\sqrt{3}}{a} \boldsymbol{\tau}_{3} .
\end{aligned}
$$

This shows that the displacements involve only the change in the bond length. The bond-length changes are

$$
\begin{aligned}
& \delta u(1)=2|\alpha| \sin \varphi \\
& \delta u(2)=2|\alpha| \sin \left(\varphi+\frac{2 \pi}{3}\right), \\
& \delta u(3)=2|\alpha| \sin \left(\varphi-\frac{2 \pi}{3}\right) .
\end{aligned}
$$

We have

$$
\delta u(1)^{2}+\delta u(2)^{2}+\delta u(3)^{2}=6|\alpha|^{2},
$$

independent of phase $\varphi$.

In a similar manner we can show that the bondlength changes around $\boldsymbol{R}_{B}=\boldsymbol{a}$ are given by those at $\boldsymbol{R}_{B}=0$ by the cyclic permutation $[\delta u(1), \delta u(2), \delta u(3)] \rightarrow$ $[\delta u(3), \delta u(1), \delta u(2)]$. Further, the bond-length changes at $\boldsymbol{R}_{B}=2 \boldsymbol{a}$ are given by those at $\boldsymbol{R}_{B}=\boldsymbol{a}$ by the cyclic permutation $[\delta u(3), \delta u(1), \delta u(2)] \rightarrow[\delta u(2), \delta u(3), \delta u(1)]$. Because $\mathrm{e}^{\mathrm{i} \boldsymbol{K} \cdot 3 \boldsymbol{a}}=\mathrm{e}^{\mathrm{i} \boldsymbol{K}^{\prime} \cdot 3 \boldsymbol{a}}=1$, the displacements at $\boldsymbol{R}_{B}=3 \boldsymbol{a}$ are the same as those at $\boldsymbol{R}_{B}=0$. Further, the relations $\mathrm{e}^{\mathrm{i} \boldsymbol{K} \cdot(2 \boldsymbol{a}+\boldsymbol{b})}=\mathrm{e}^{\mathrm{i} \boldsymbol{K}^{\prime} \cdot(2 \boldsymbol{a}+\boldsymbol{b})}=1$ and $\mathrm{e}^{\mathrm{i} \boldsymbol{K} \cdot(\boldsymbol{a}+2 \boldsymbol{b})}=$ $\mathrm{e}^{\mathrm{i} \boldsymbol{K}^{\prime} \cdot(\boldsymbol{a}+2 \boldsymbol{b})}=1$ show that the displacements at $\boldsymbol{R}_{B}=$ $-\boldsymbol{b}$ are the same as those at $\boldsymbol{R}_{B}=2 \boldsymbol{a}$ and that the displacements at $\boldsymbol{R}_{B}=-2 \boldsymbol{b}$ are the same as those at $\boldsymbol{R}_{B}=\boldsymbol{a}$. In this way, we can demonstrate that these are Kekule distortions reducing the Brillouin-zone area by a factor of three. The specific Kekulé distortion considered previously $^{62-64)}$ corresponds to $\varphi= \pm \pi / 2$ and $u_{1}= \pm|\alpha|$, where $u_{1}$ has been defined in ref. 62 .

\subsection{Interaction Hamiltonian}

In the presence of lattice displacement, the hopping integral between an atom at $\boldsymbol{R}_{A}$ and an atom at $\boldsymbol{R}_{A}-\boldsymbol{\tau}_{l}$ becomes

$$
\begin{aligned}
& \gamma_{0}\left(\left|\boldsymbol{\tau}_{l}+\boldsymbol{u}_{A}\left(\boldsymbol{R}_{A}\right)-\boldsymbol{u}_{B}\left(\boldsymbol{R}_{A}-\boldsymbol{\tau}_{l}\right)\right|\right) \\
& =\gamma_{0}+\frac{1}{b} \frac{\partial \gamma_{0}}{\partial b} \boldsymbol{\tau}_{l} \cdot\left[\boldsymbol{u}_{A}\left(\boldsymbol{R}_{A}\right)-\boldsymbol{u}_{B}\left(\boldsymbol{R}_{A}-\boldsymbol{\tau}_{l}\right)\right]
\end{aligned}
$$

Similar expression is readily obtained for the hopping integral between an atom at $\boldsymbol{R}_{B}$ and an atom at $\boldsymbol{R}_{B}+\boldsymbol{\tau}_{l}$. Then, the effective-mass Hamiltonian in the presence of the displacement corresponding to phonons at the $\mathrm{K}$ and $\mathrm{K}$ ' points can be calculated as described in Appendix A. The resulting Hamiltonian shows that only mode $g=1$ contributes to the interaction.

Let us define

$$
e_{K}=e_{1}^{K}, \quad e_{K^{\prime}}=e_{1}^{K^{\prime}}
$$

and

$$
U_{K}(\boldsymbol{r})=\left(\begin{array}{c}
\boldsymbol{u}_{A}^{K}(\boldsymbol{r}) \\
\boldsymbol{u}_{B}^{K}(\boldsymbol{r})
\end{array}\right), \quad \boldsymbol{U}_{K^{\prime}}(\boldsymbol{r})=\left(\begin{array}{c}
\boldsymbol{u}_{A}^{K^{\prime}}(\boldsymbol{r}) \\
\boldsymbol{u}_{B}^{K^{\prime}}(\boldsymbol{r})
\end{array}\right) .
$$

Define, further

$$
\Delta_{K}(\boldsymbol{r})=\boldsymbol{e}_{K}^{\dagger} \boldsymbol{U}_{K}(\boldsymbol{r}), \quad \Delta_{K^{\prime}}(\boldsymbol{r})=\boldsymbol{e}_{K^{\prime}}^{\dagger} \boldsymbol{U}_{K^{\prime}}(\boldsymbol{r}),
$$

which give

$$
\begin{aligned}
& \Delta_{K}(\boldsymbol{r})=\sum_{\boldsymbol{q}} \sqrt{\frac{\hbar}{2 N M \omega_{K}}}\left(b_{K \boldsymbol{q}}+b_{K^{\prime}-\boldsymbol{q}}^{\dagger}\right) \mathrm{e}^{\mathrm{i} \boldsymbol{q} \cdot \boldsymbol{r}}, \\
& \Delta_{K^{\prime}}(\boldsymbol{r})=\sum_{\boldsymbol{q}} \sqrt{\frac{\hbar}{2 N M \omega_{K}}}\left(b_{K^{\prime} \boldsymbol{q}}+b_{K-\boldsymbol{q}}^{\dagger}\right) \mathrm{e}^{\mathrm{i} \boldsymbol{q} \cdot \boldsymbol{r}},
\end{aligned}
$$

where $\omega_{K}=\omega_{1}$ and $b_{K \boldsymbol{q}}=b_{K, 1, \boldsymbol{q}}, b_{K^{\prime} \boldsymbol{q}}=b_{K^{\prime}, 1, \boldsymbol{q}}$, etc. Obviously, we have

$$
\Delta_{K}(\boldsymbol{r})^{*}=\Delta_{K^{\prime}}(\boldsymbol{r})
$$

Then, the Hamiltonian is given by

$$
\mathcal{H}_{\text {int }}=2 \frac{\beta_{K} \gamma}{b^{2}}\left(\begin{array}{cc}
0 & \omega^{-1} \Delta_{K^{\prime}}(\boldsymbol{r}) \sigma_{y} \\
\omega \Delta_{K}(\boldsymbol{r}) \sigma_{y} & 0
\end{array}\right)
$$

where

$$
\beta_{K}=-\frac{\mathrm{d} \ln \gamma_{0}}{\mathrm{~d} \ln b}=-\frac{b}{\gamma_{0}} \frac{\mathrm{d} \gamma_{0}}{\mathrm{~d} b} .
$$

This interaction Hamiltonian has been obtained within the valence-force-field model and the nearestneighbor tight-binding model, but is expected to be much more general if $\beta_{K}$ is regarded as an appropriate parameter. For a uniform distortion $\alpha=\left\langle\Delta_{K^{\prime}}(\boldsymbol{r})\right\rangle$ and $\alpha^{*}=\left\langle\Delta_{K}(\boldsymbol{r})\right\rangle$, this Hamiltonian is equivalent to that derived previously within a phase factor, ${ }^{62)}$ which can be removed by an appropriate phase change of the wave function $\boldsymbol{F}^{K}(\boldsymbol{r})$ and $\boldsymbol{F}^{K^{\prime}}(\boldsymbol{r})$ or of $\Delta_{K}(\boldsymbol{r})$ and $\Delta_{K^{\prime}}(\boldsymbol{r})$.

Because only the "Kekulé mode" $g=1$ couples with electrons in the vicinity of the $\mathrm{K}$ and $\mathrm{K}$ ' points, we shall consider this mode only and suppress the mode index $g$ in the following. By the $\mathrm{K}$ phonon, we shall mean the mode $g=1$.

\subsection{Zone-Center Phonon}

For comparison, we shall give a brief review on long-wavelength phonons near the $\Gamma$ point. First, we shall consider optical phonons. ${ }^{2,3)}$ Let $\boldsymbol{u}^{\mathrm{op}}=\left(u_{x}^{\mathrm{op}}, u_{y}^{\mathrm{op}}\right)$ 
be the relative displacement of two sub-lattice atoms $\mathrm{A}$ and B, i.e.,

$$
\boldsymbol{u}^{\mathrm{op}}(\boldsymbol{R})=\frac{1}{\sqrt{2}}\left[\boldsymbol{u}_{A}(\boldsymbol{R})-\boldsymbol{u}_{B}(\boldsymbol{R})\right]
$$

where $\boldsymbol{R}$ denotes a coordinate specifying a unit cell. In the long-wavelength limit $\boldsymbol{R}$ can be replaced by a continuous coordinate $\boldsymbol{r}$. Then, we have

$$
\boldsymbol{u}^{\mathrm{op}}(\boldsymbol{r})=\sum_{\boldsymbol{q}, g} \sqrt{\frac{\hbar}{2 N M \omega_{0}}}\left(b_{\boldsymbol{q} g}+b_{-\boldsymbol{q} g}^{\dagger}\right) \boldsymbol{e}_{g}(\boldsymbol{q}) \mathrm{e}^{\mathrm{i} \boldsymbol{q} \cdot \boldsymbol{r}}
$$

where $\omega_{0}$ is the phonon frequency at the $\Gamma$ point and $g$ denotes the modes ( $\mathrm{t}$ for transverse and $\mathrm{l}$ for longitudinal). Define

$$
q_{x}=q \cos \varphi(\boldsymbol{q}), \quad q_{y}=q \sin \varphi(\boldsymbol{q}),
$$

with $q=|\boldsymbol{q}|$. Then, we have

$$
\begin{aligned}
& \boldsymbol{e}_{\mathrm{l}}(\boldsymbol{q})=\mathrm{i}(\cos \varphi(\boldsymbol{q}), \sin \varphi(\boldsymbol{q})), \\
& \boldsymbol{e}_{\mathrm{t}}(\boldsymbol{q})=\mathrm{i}(-\sin \varphi(\boldsymbol{q}), \cos \varphi(\boldsymbol{q})) .
\end{aligned}
$$

The interaction Hamiltonian becomes

$$
\mathcal{H}_{\mathrm{int}}=-\sqrt{2} \frac{\beta_{\Gamma} \gamma}{b^{2}}\left(\begin{array}{cc}
\boldsymbol{\sigma} \times \boldsymbol{u}^{\mathrm{op}}(\boldsymbol{r}) & 0 \\
0 & -\boldsymbol{\sigma}^{*} \times \boldsymbol{u}^{\mathrm{op}}(\boldsymbol{r})
\end{array}\right) .
$$

where the vector product for vectors $\boldsymbol{a}=\left(a_{x}, a_{y}\right)$ and $\boldsymbol{b}=$ $\left(b_{x}, b_{y}\right)$ in two dimension is defined by $\boldsymbol{a} \times \boldsymbol{b}=a_{x} b_{y}-a_{y} b_{x}$. The dimensionless parameter $\beta_{\Gamma}$ is the same as $\beta_{K}$ in the nearest-neighbor tight-binding model, but can be slightly different for $\beta_{K}$ in a more elaborate model.

The long-wavelength acoustic phonons can be described by an elastic continuum model. ${ }^{1)}$ The interaction is usually dominated by the deformation potential

$$
\mathcal{H}_{1}=g_{1}\left(\begin{array}{cc}
u_{x x}(\boldsymbol{r})+u_{y y}(\boldsymbol{r}) & 0 \\
0 & u_{x x}(\boldsymbol{r})+u_{y y}(\boldsymbol{r})
\end{array}\right),
$$

where $g_{1}$ is the deformation potential constant, known to be about $16 \mathrm{eV}$ in bulk graphite ${ }^{65)}$ and estimated to be 20-30 eV within a two-dimensional free-electron model, ${ }^{1)}$ and the strain tensor is given by

$$
u_{x x}=\frac{\partial u_{x}^{\mathrm{ac}}}{\partial x}, u_{y y}=\frac{\partial u_{y}^{\mathrm{ac}}}{\partial y}, u_{x y}=\frac{1}{2}\left(\frac{\partial u_{x}^{\mathrm{ac}}}{\partial y}+\frac{\partial u_{y}^{\mathrm{ac}}}{\partial x}\right),
$$

where $\boldsymbol{u}^{\text {ac }}(\boldsymbol{r})$ represents the lattice displacement for the center of mass of two sub-lattice atoms,

$$
\boldsymbol{u}^{\mathrm{ac}}(\boldsymbol{R})=\frac{1}{\sqrt{2}}\left[\boldsymbol{u}_{A}(\boldsymbol{R})+\boldsymbol{u}_{B}(\boldsymbol{R})\right]
$$

In carbon nanotubes, an additional term $u_{z}^{\text {ac }} / R$ should be added to $u_{x x}$ due to curvature when the $x$ is chosen in the circumference direction, where $u_{z}^{\text {ac }}(\boldsymbol{r})$ is the out-of-plane distortion and $R$ is the radius given by $R=L / 2 \pi$.

A long-wavelength acoustic phonon gives rise to a small change in the bond length and therefore modifies the band structure. This effect gives a small coupling described by the Hamiltonian

$$
\mathcal{H}_{2}=g_{2}\left(\begin{array}{cc}
\sigma_{x} \operatorname{Re} V_{2}-\sigma_{y} \operatorname{Im} V_{2} & 0 \\
0 & -\sigma_{x} \operatorname{Re} V_{2}-\sigma_{y} \operatorname{Im} V_{2}
\end{array}\right),
$$

with

$$
V_{2}=\mathrm{e}^{3 i \eta}\left(u_{x x}-u_{y y}+2 i u_{x y}\right),
$$

and coupling parameter

$$
g_{2}=\frac{\kappa \beta_{\Gamma} \gamma}{2 b}
$$

where $\kappa \approx 1 / 3$ in the valence-force-field model. ${ }^{1)}$ The parameter $g_{2} \approx \beta_{\Gamma} \gamma_{0} / 4$ is much smaller than $g_{1}$ for $\gamma_{0} \sim 3$ $\mathrm{eV}$ and $\beta_{\Gamma} \sim 2$. Therefore, this Hamiltonian $\mathcal{H}_{2}$ can usually be neglected except in a special case of metallic nanotubes for which the absence of backscattering ${ }^{42,44)}$ plays important roles due to the presence of a special time-reversal symmetry as will briefly discussed below.

\subsection{Symmetry Properties}

Under the time-reversal operation $\mathcal{T}$, the $\mathrm{K}$ and $\mathrm{K}^{\prime}$ points are exchanged corresponding to the complex conjugate wave functions. In the present $\boldsymbol{k} \cdot \boldsymbol{p}$ scheme, the $\mathcal{T}$ operation gives $\boldsymbol{F}^{K T}=\mathrm{e}^{-\mathrm{i} \psi} \sigma_{z} \boldsymbol{F}^{K^{\prime} *}$ and $\boldsymbol{F}^{K^{\prime} T}=$ $\mathrm{e}^{-\mathrm{i} \psi} \sigma_{z} \boldsymbol{F}^{K *}$, where $*$ denotes the complex conjugate, $\psi$ is an arbitrary phase, and $\sigma_{z}$ is a Pauli spin matrix. ${ }^{9,49)}$ The derivation is given in Appendix of ref. 66 and discussed also in Appendix B. It is straightforward to demonstrate that the Hamiltonian including interaction with acoustic and optical phonons and zone-boundary phonons is invariant under this $\mathcal{T}$ operation.

Within each $\mathrm{K}$ and $\mathrm{K}$ ' points, we can define a special time-reversal operation $\mathcal{S}$ such that $\boldsymbol{F}^{S}=K \boldsymbol{F}^{*}$ with $K=-\mathrm{i} \sigma_{y}$, where $\boldsymbol{F}$ is $\boldsymbol{F}^{K}$ or $\boldsymbol{F}^{K^{\prime}}$. This corresponds to the usual time-reversal operation in the presence of spin-orbit interaction. When intervalley scattering between $\mathrm{K}$ and $\mathrm{K}$ ' points is not important, this $\mathcal{S}$ symmetry plays a dominant role and the real time-reversal symmetry $\mathcal{T}$ becomes irrelevant. In fact, most potential scatterers including charged centers are characterized by a potential with range larger than the lattice constant. For such scatterers, the same potential appears as the diagonal element of the matrix Hamiltonian and mixing between $\mathrm{K}$ and $\mathrm{K}^{\prime}$ points is safely neglected.

When $\mathcal{S}$ is operated twice, the wave function changes its sign, i.e., $\boldsymbol{F}^{S^{2}}=\left(\boldsymbol{F}^{S}\right)^{S}=-\boldsymbol{F}$. The corresponding operation for operator $P$ is $P^{S}=K^{t} P K^{-1}$, where ${ }^{t} P$ stands for the transpose of $P$. We have $\left(\boldsymbol{F}_{\alpha}^{S}, P^{S} \boldsymbol{F}_{\beta}^{S}\right)=$ $\left(\boldsymbol{F}_{\beta}, P \boldsymbol{F}_{\alpha}\right)$. For a diagonal impurity potential $V(\boldsymbol{r})$, obviously we have $V(\boldsymbol{r})^{S}=V(\boldsymbol{r})$. Let $r_{\bar{\beta} \alpha}$ be the reflection coefficient from a state with wave vector $\mathbf{k}_{\alpha}$ to a state with $\mathbf{k}_{\bar{\beta}} \equiv-\mathbf{k}_{\beta}$. Here, $\bar{\beta}$ stands for the state with wave vector opposite to $\beta$. We have $r_{\bar{\beta} \alpha}=$ $\left(\mathbf{F}_{\beta}^{S}, T \mathbf{F}_{\alpha}\right)$ in terms of the so-called $\mathrm{T}$ matrix, given by $T=V+V G_{0} V+V G_{0} V G_{0} V+\cdots$, where $G_{0}$ is the Green's function in the absence of scatterers. The $T$ matrix satisfies $T^{S}=T$. With the use of the $\mathcal{S}$ symmetry, we have $\left(\mathbf{F}_{\beta}^{S}, T \mathbf{F}_{\alpha}\right)=\left(\mathbf{F}_{\alpha}^{S}, T \mathbf{F}_{\beta}^{S^{2}}\right)=-\left(\mathbf{F}_{\alpha}^{S}, T \mathbf{F}_{\beta}\right)$, leading to the symmetry relation $r_{\bar{\beta} \alpha}=-r_{\bar{\alpha} \beta} \cdot{ }^{45)}$ This immediately gives the absence of backscattering $r_{\bar{\alpha} \alpha}=0$.

The deformation potential $\mathcal{H}_{1}$ given by eq. (2.59) is 
invariant under the $\mathcal{S}$ operation like impurity potential and therefore cannot cause backscattering in graphene. This leads to the absence of backscattering in metallic nanotubes. In semiconducting nanotubes, the scattering between states with the same absolute value of the wave vector in the axis direction is possible, because it does not correspond to exact backscattering in graphene. On the other hand, the interaction Hamiltonians arising from the bond length change, such as $\mathcal{H}_{2}$ given by eq. (2.62) for acoustic modes and $\mathcal{H}_{\text {int }}$ given by eq. (2.58) for optical modes, destroy the $\mathcal{S}$ symmetry and therefore cause backscattering. The Hamiltonian for the interaction with the zone-boundary phonon causes intervalley mixing, making the $\mathcal{S}$ symmetry irrelevant, and therefore causes backscattering.

The Hamiltonians (2.52) for zone-boundary phonons and (2.58) for zone-center optical phonons are independent of the chiral angle $\eta$ within the approximation neglecting the dispersions of phonons. The deformation potential (2.59) is also independent of $\eta$. Only the small correction $\mathcal{H}_{2}$ depends on the chirality of a nanotube. Because of the absence of backscattering for the deformation potential, the resistance in metallic nanotubes is dominated by chirality-dependent $\mathcal{H}_{2}$ as long as the Fermi level lies in the linear bands. In graphene, semiconducting nanotubes, and metallic nanotubes with the Fermi level in higher bands, the interaction is dominated by chirality-independent $\mathcal{H}_{1}$.

In the case of zone-center optical phonons and zoneboundary phonons, no singular property like the absence of backscattering is present and therefore the lowest order Hamiltonian independent of the chirality works sufficiently well. To higher orders in the wave vector, various chirality-dependent corrections can appear in electron-phonon interaction, but are expected to cause only a small modification of the results. Direct higherorder corrections for electronic states, such as trigonal warping, ${ }^{67,48)}$ are likely to be much more important than those for the electron-phonon interactions.

\section{$\S 3$. Electron-Phonon Scattering in Graphene}

In graphene, the states are specified by the band index $s= \pm 1$ ( $s=+1$ for the conduction band and -1 for the valence band) and the wave vector $\boldsymbol{k}$. The wave functions are given by

$$
\boldsymbol{F}_{s, \boldsymbol{k}}^{K}(\boldsymbol{r})=\frac{1}{L} \mathrm{e}^{\mathrm{i} \boldsymbol{k} \cdot \boldsymbol{r}} \boldsymbol{F}_{s, \boldsymbol{k}}^{K}, \quad \boldsymbol{F}_{s, \boldsymbol{k}}^{K^{\prime}}(\boldsymbol{r})=\frac{1}{L} \mathrm{e}^{\mathrm{i} \boldsymbol{k} \cdot \boldsymbol{r}} \boldsymbol{F}_{s, \boldsymbol{k}}^{K^{\prime}},
$$

with

$$
\boldsymbol{F}_{s, \boldsymbol{k}}^{K}=\frac{1}{\sqrt{2}}\left(\begin{array}{c}
s \\
\mathrm{e} \mathrm{i} \theta(\boldsymbol{k})
\end{array}\right), \quad \boldsymbol{F}_{s, \boldsymbol{k}}^{K^{\prime}}=\frac{1}{\sqrt{2}}\left(\begin{array}{c}
\mathrm{e}^{\mathrm{i} \theta(\boldsymbol{k})} \\
s
\end{array}\right),
$$

where

$$
k_{x}=k \cos \theta(\boldsymbol{k}), \quad k_{y}=k \sin \theta(\boldsymbol{k}), \quad k=|\boldsymbol{k}| .
$$

The corresponding energies are given by

$$
\varepsilon_{s, \boldsymbol{k}}=s \gamma k \text {. }
$$

We have

$$
\left|\left(K^{\prime} s^{\prime} \boldsymbol{k}^{\prime}\left|\sigma_{y}\right| K s \boldsymbol{k}\right)\right|^{2}=\frac{1}{2}\left(1-s s^{\prime} \cos \left[\theta(\boldsymbol{k})-\theta\left(\boldsymbol{k}^{\prime}\right)\right]\right) \text {. }
$$

The probability of an electron at the $\mathrm{K}$ point being scattered into the $\mathrm{K}^{\prime}$ point by emitting and absorbing a phonon is

$$
\begin{aligned}
\frac{\hbar}{\tau}=2 \pi\left(\frac{2 \beta_{K} \gamma}{b^{2}}\right)^{2} \frac{\hbar L^{2}}{2 N M \omega_{K}} & \frac{1}{2} \frac{1}{2 \pi \gamma^{2}}\left[n_{K}\left|\varepsilon+\hbar \omega_{K}\right|\right. \\
& \left.+\left(n_{K}+1\right)\left|\varepsilon-\hbar \omega_{K}\right|\right]
\end{aligned}
$$

where $n_{K}$ is the Planck distribution function for phonon with frequency $\omega_{K}$.

Define the dimensionless coupling parameter $\lambda_{K}$ as

$$
\lambda_{K}=\frac{36 \sqrt{3}}{\pi} \frac{\hbar^{2}}{2 M a^{2}} \frac{1}{\hbar \omega_{K}}\left(\frac{\beta_{K}}{2}\right)^{2} .
$$

For $\hbar \omega_{K}=161.2 \mathrm{meV}$ and $\gamma_{0}=2.63 \mathrm{eV}$, this becomes

$$
\lambda_{K}=3.5 \times 10^{-3}\left(\frac{\beta_{K}}{2}\right)^{2} .
$$

Then, we have

$$
\frac{\hbar}{\tau}=\pi \lambda_{K}\left[n_{K}\left|\varepsilon+\hbar \omega_{K}\right|+\left(n_{K}+1\right)\left|\varepsilon-\hbar \omega_{K}\right|\right] .
$$

The typical scattering strength can be obtained by considering the emission probability at low temperature, i.e., $n_{K}=0$,

$$
\frac{\hbar}{\tau_{0}}=\pi \lambda_{K}\left|\varepsilon-\hbar \omega_{K}\right|
$$

For comparison, we shall calculate the scattering probability for optical phonons at the $\Gamma$ point. The sum of the contributions of the longitudinal and transverse modes gives isotropic scattering probability,

$$
\frac{\hbar}{\tau}=\pi \lambda_{\Gamma}\left[n_{\Gamma}\left|\varepsilon+\hbar \omega_{\Gamma}\right|+\left(n_{\Gamma}+1\right)\left|\varepsilon-\hbar \omega_{\Gamma}\right|\right]
$$

where $\lambda_{\Gamma}$ is the dimensionless coupling constant

$$
\lambda_{\Gamma}=\frac{36 \sqrt{3}}{\pi} \frac{\hbar^{2}}{2 M a^{2}} \frac{1}{\hbar \omega_{\Gamma}}\left(\frac{\beta_{\Gamma}}{2}\right)^{2} .
$$

For $\hbar \omega_{\Gamma}=196 \mathrm{meV}$, this becomes

$$
\lambda_{\Gamma}=2.9 \times 10^{-3}\left(\frac{\beta_{\Gamma}}{2}\right)^{2} .
$$

The emission probability at low temperatures becomes

$$
\frac{\hbar}{\tau_{0}}=\pi \lambda_{\Gamma}\left|\varepsilon-\hbar \omega_{\Gamma}\right|
$$

The above shows that the scattering by phonons at the $\mathrm{K}$ and $\mathrm{K}$ ' points is stronger than that at the $\Gamma$ point. At or below room temperatures, the number of excited optical phonons at the $\Gamma$ point and phonons at the $\mathrm{K}$ and $\mathrm{K}$ ' points are small and therefore the emission process is dominant. Usually, the emission becomes possible when the electron energy exceeds the phonon energy, because otherwise the final states are occupied by electrons at low temperature. This means that the K-phonon scattering is much more dominant than the $\Gamma$-phonon scattering in 
the high-field transport because of the larger coupling constant and the lower phonon energy.

\section{$\S 4$. Electron-Phonon Scattering in Nanotube}

\subsection{Zone-Boundary Phonon}

In nanotubes with chiral vector $\boldsymbol{L}=n_{a} \boldsymbol{a}+n_{b} \boldsymbol{b}$, the allowed phonon modes should satisfy the periodic boundary conditions:

$$
\boldsymbol{u}_{A}\left(\boldsymbol{R}_{A}+\boldsymbol{L}\right)=\boldsymbol{u}_{A}\left(\boldsymbol{R}_{A}\right), \quad \boldsymbol{u}_{B}\left(\boldsymbol{R}_{B}+\boldsymbol{L}\right)=\boldsymbol{u}_{B}\left(\boldsymbol{R}_{B}\right) .
$$

In the way analogous to the case of electrons, ${ }^{5}$ ) they lead to the following boundary conditions for the slowlyvarying part:

$$
\begin{gathered}
\boldsymbol{u}_{A}^{K}(\boldsymbol{r}+\boldsymbol{L})=\boldsymbol{u}_{A}^{K}(\boldsymbol{r}) \mathrm{e}^{-\mathrm{i} \boldsymbol{K} \cdot \boldsymbol{L}}=\boldsymbol{u}_{A}^{K}(\boldsymbol{r}) \exp \left(-\frac{2 \pi \mathrm{i} \nu}{3}\right), \\
\boldsymbol{u}_{B}^{K}(\boldsymbol{r}+\boldsymbol{L})=\boldsymbol{u}_{B}^{K}(\boldsymbol{r}) \mathrm{e}^{-\mathrm{i} \boldsymbol{K} \cdot \boldsymbol{L}}=\boldsymbol{u}_{B}^{K}(\boldsymbol{r}) \exp \left(-\frac{2 \pi \mathrm{i} \nu}{3}\right), \\
\boldsymbol{u}_{A}^{K^{\prime}}(\boldsymbol{r}+\boldsymbol{L})=\boldsymbol{u}_{A}^{K^{\prime}}(\boldsymbol{r}) \mathrm{e}^{-\mathrm{i} \boldsymbol{K}^{\prime} \cdot \boldsymbol{L}}=\boldsymbol{u}_{A}^{K^{\prime}}(\boldsymbol{r}) \exp \left(+\frac{2 \pi \mathrm{i} \nu}{3}\right), \\
\boldsymbol{u}_{B}^{K^{\prime}}(\boldsymbol{r}+\boldsymbol{L})=\boldsymbol{u}_{B}^{K^{\prime}}(\boldsymbol{r}) \mathrm{e}^{-\mathrm{i} K^{\prime} \cdot \boldsymbol{L}}=\boldsymbol{u}_{B}^{K^{\prime}}(\boldsymbol{r}) \exp \left(+\frac{2 \pi \mathrm{i} \nu}{3}\right),
\end{gathered}
$$

where $\nu=0$ and \pm 1 , given by

$$
n_{a}+n_{b}=3 m+\nu,
$$

with integer $m$. Therefore, the allowed wave vector in the circumference direction becomes discrete,

$$
\begin{aligned}
& q_{x}= \begin{cases}\kappa_{\nu}(j) & \text { (for the } \mathrm{K} \text { point) }, \\
\kappa_{-\nu}(j) & \text { (for the } \mathrm{K}^{\prime} \text { point), }\end{cases} \\
& \kappa_{\nu}(j)=\frac{2 \pi}{L}\left(j-\frac{\nu}{3}\right),
\end{aligned}
$$

with $j$ being an integer, but the wave vector in the axis direction $q$ remains continuous. Note that $\kappa_{-\nu}(j)=$ $-\kappa_{\nu}(-j)$. Therefore, the displacement is written as

$$
\begin{gathered}
\Delta_{K}(\boldsymbol{r})=\sum_{j, q} \sqrt{\frac{\hbar}{2 N M \omega_{K}}}\left(b_{K, j, q}+b_{K^{\prime},-j,-q}^{\dagger}\right) \\
\times \exp \left[\mathrm{i} \kappa_{\nu}(j) x+\mathrm{i} q y\right], \\
\Delta_{K^{\prime}}(\boldsymbol{r})=\sum_{j, q} \sqrt{\frac{\hbar}{2 N M \omega_{K}}}\left(b_{K^{\prime}, j, q}+b_{K,-j,-q}^{\dagger}\right) \\
\times \exp \left[\mathrm{i} \kappa_{-\nu}(j) x+\mathrm{i} q y\right],
\end{gathered}
$$

where $N$ is the total number of unit cells in the nanotube.

\subsection{Electronic states}

The electronic states are specified by the band index $s= \pm 1$, the integer $n$ specifying the wave vector in the circumference direction, and wave vector $k$ in the axis direction. The wave functions are written as

$$
\begin{aligned}
& \boldsymbol{F}_{s, n, k}^{K}(\boldsymbol{r})=\frac{1}{\sqrt{L A}} \exp \left[\mathrm{i} \kappa_{\nu}(n) x+\mathrm{i} k y\right] \boldsymbol{F}_{s, n, k}^{K}, \\
& \boldsymbol{F}_{s, n, k}^{K^{\prime}}(\boldsymbol{r})=\frac{1}{\sqrt{L A}} \exp \left[\mathrm{i} \kappa_{-\nu}(n) x+\mathrm{i} k y\right] \boldsymbol{F}_{s, n, k}^{K^{\prime}},
\end{aligned}
$$

with

$\boldsymbol{F}_{s, n, k}^{K}=\frac{1}{\sqrt{2}}\left(\begin{array}{c}b_{\nu}(n, k) \\ s\end{array}\right), \quad \boldsymbol{F}_{s, n, k}^{K^{\prime}}=\frac{1}{\sqrt{2}}\left(\begin{array}{c}b_{-\nu}(n, k)^{*} \\ s\end{array}\right)$, where $A$ is the length of the nanotube and

$$
b_{\nu}(n, k)=\frac{\kappa_{\nu}(n)-\mathrm{i} k}{\sqrt{\kappa_{\nu}(n)^{2}+k^{2}}} .
$$

The corresponding energies are

$$
\varepsilon_{s, n, k}^{K}=s \gamma \sqrt{\kappa_{\nu}(n)^{2}+k^{2}}, \quad \varepsilon_{s, n, k}^{K^{\prime}}=s \gamma \sqrt{\kappa_{-\nu}(n)^{2}+k^{2}} .
$$

Figure 3 shows a schematic illustration of scattering process by emitting and absorbing a phonon. We have the matrix element

$$
\begin{aligned}
& \left|\boldsymbol{F}_{s^{\prime}, n^{\prime}, k^{\prime}}^{K^{\prime} \dagger} \sigma_{y} \boldsymbol{F}_{s, n, k}^{K}\right|^{2}=\frac{1}{2}\left\{1-s^{\prime} s \operatorname{Re}\left[b_{-\nu}\left(n^{\prime}, k^{\prime}\right)^{*} b_{\nu}(n, k)\right]\right\} \\
& =\frac{1}{2}\left(1-s^{\prime} s \frac{\kappa_{-\nu}\left(n^{\prime}\right) \kappa_{\nu}(n)+k^{\prime} k}{\sqrt{\kappa_{-\nu}\left(n^{\prime}\right)^{2}+k^{\prime 2}} \sqrt{\kappa_{\nu}(n)^{2}+k^{2}}}\right) .
\end{aligned}
$$

Further, we have

$$
\begin{aligned}
& \frac{1}{L} \int_{-L / 2}^{L / 2} \mathrm{~d} x \exp \left[-\mathrm{i} \kappa_{-\nu}\left(n^{\prime}\right) x\right] \exp \left[\mathrm{i} \kappa_{\nu}(j) x\right] \exp \left[\mathrm{i} \kappa_{\nu}(n) x\right] \\
& =\delta_{n^{\prime}, n+j-\nu}
\end{aligned}
$$

Therefore, the scattering probability becomes

$$
\begin{aligned}
& \frac{\hbar}{\tau}=2 \pi\left(\frac{2 \beta_{K} \gamma}{b^{2}}\right)^{2} \frac{\hbar}{2 N M \omega_{K}} \sum_{s^{\prime} n^{\prime}, k^{\prime}} \sum_{j, q}\left|\boldsymbol{F}_{s^{\prime}, n^{\prime}, k^{\prime}}^{K^{\prime} \dagger} \sigma_{y} \boldsymbol{F}_{s, n, k}^{K}\right|^{2} \\
& \times \delta_{k^{\prime}, k+q} \delta_{n^{\prime}, n+j-\nu}\left[n_{K} \delta\left(\varepsilon_{s, n, k}^{K}-\varepsilon_{s^{\prime}, n^{\prime}, k^{\prime}}^{K^{\prime}}+\hbar \omega_{K}\right)\right. \\
&\left.+\left(n_{K}+1\right) \delta\left(\varepsilon_{s, n, k}^{K}-\varepsilon_{s^{\prime}, n^{\prime}, k^{\prime}}^{K}-\hbar \omega_{K}\right)\right] .
\end{aligned}
$$

After the summation over $j$ and $q$, we have

$$
\begin{aligned}
\frac{\hbar}{\tau}=2 \pi & \left(\frac{2 \beta_{K} \gamma}{b^{2}}\right)^{2} \frac{\hbar}{2 N M \omega_{K}} \sum_{s^{\prime} n^{\prime}, k^{\prime}}\left|\boldsymbol{F}_{s^{\prime}, n^{\prime}, k^{\prime}}^{K^{\prime} \dagger} \sigma_{y} \boldsymbol{F}_{s, n, k}^{K}\right|^{2} \\
& \times\left[n_{K} \delta\left(\varepsilon_{s, n, k}^{K}-\varepsilon_{s^{\prime}, n^{\prime}, k^{\prime}}^{K^{\prime}}+\hbar \omega_{K}\right)\right. \\
& \left.+\left(n_{K}+1\right) \delta\left(\varepsilon_{s, n, k}^{K}-\varepsilon_{s^{\prime}, n^{\prime}, k^{\prime}}^{K^{\prime}}-\hbar \omega_{K}\right)\right] .
\end{aligned}
$$

\subsection{Semiconducting nanotube}

First, we consider the case of the lowest conduction band $(s=+1, n=0$, and $k>0)$ in a semiconducting nanotube as illustrated in the right part of Fig. 3. Except in very thick nanotubes, interband scattering can be safely neglected and we can limit ourselves to the case of $s^{\prime}=+1$ and $n^{\prime}=0$. Then we have

$$
\begin{aligned}
\frac{\hbar}{\tau}=\lambda_{K} \frac{2 \pi \gamma}{L} \sum_{ \pm}\left[\frac{1}{2}(\right. & \left.1+\frac{\kappa^{2}-\left( \pm k_{+}\right) k}{\left[\left(\kappa^{2}+k_{+}^{2}\right)\left(\kappa^{2}+k^{2}\right)\right]^{1 / 2}}\right) \\
& \times n_{K} n\left(\varepsilon+\hbar \omega_{K}\right) \\
+ & \frac{1}{2}\left(1+\frac{\kappa^{2}-\left( \pm k_{-}\right) k}{\left[\left(\kappa^{2}+k_{-}^{2}\right)\left(\kappa^{2}+k^{2}\right)\right]^{1 / 2}}\right) \\
& \left.\times\left(n_{K}+1\right) n\left(\varepsilon-\hbar \omega_{K}\right)\right],
\end{aligned}
$$

with

$$
\kappa \equiv \frac{2 \pi}{L} \frac{\nu}{3}
$$

where $\varepsilon=\varepsilon_{+, 0, k}$, the summation \pm means that over $+k_{ \pm}$ 
and $-k_{ \pm}$satisfying the energy conservation condition

$$
\gamma \sqrt{\kappa^{2}+k_{ \pm}^{2}}=\varepsilon \pm \hbar \omega_{K}
$$

and

$$
n(\varepsilon)=\frac{\varepsilon}{\sqrt{\varepsilon^{2}-\varepsilon_{0}^{2}}} \theta\left(\varepsilon-\varepsilon_{0}\right),
$$

with the energy of the band bottom given by

$$
\varepsilon_{0}=\gamma|\kappa|
$$

and $\theta(t)=1$ for $t>0$ and $\theta(t)=0$ for $t<0$.

\subsection{Metallic nanotube}

In metallic nanotubes as illustrated in the left part of Fig. 3, we have

$$
\left|\boldsymbol{F}_{s^{\prime}, n^{\prime}, k^{\prime}}^{K^{\prime} \dagger} \sigma_{y} \boldsymbol{F}_{s, n, k}^{K}\right|^{2}=\frac{1}{2}\left(1-s^{\prime} \frac{k^{\prime}}{|k|}\right)
$$

for $s=+1$ and $k>0$, showing that only backward scattering is present. Then, we have

$$
\frac{\hbar}{\tau}=\lambda_{K} \frac{2 \pi \gamma}{L}\left(2 n_{K}+1\right)
$$

Note that the result for metallic nanotubes can be obtained from that for semiconducting nanotubes by setting $\kappa=0$ and therefore $n(\varepsilon)=1$.

\subsection{Comparison with Zone-Center Phonon}

For comparison, we consider the scattering by the $\Gamma$ optical phonons. We have

$$
\left|\boldsymbol{F}_{s^{\prime}, n^{\prime}, k^{\prime}}^{K \dagger} \sigma_{x} \boldsymbol{F}_{s, n, k}^{K}\right|^{2}+\left|\boldsymbol{F}_{s^{\prime}, n^{\prime}, k^{\prime}}^{K \dagger} \sigma_{y} \boldsymbol{F}_{s, n, k}^{K}\right|^{2}=1,
$$

independent of the bands and whether the tube is metallic or semiconducting. Therefore, the scattering probability is given by

$$
\frac{\hbar}{\tau}=\frac{1}{2} \lambda_{\Gamma} \frac{2 \pi \gamma}{L} \sum_{ \pm}\left[n_{\Gamma} n\left(\varepsilon+\hbar \omega_{\Gamma}\right)+\left(n_{\Gamma}+1\right) n\left(\varepsilon-\hbar \omega_{\Gamma}\right)\right]
$$

where $n(\varepsilon)=1$ in the metallic case.

The typical values of the scattering probability for the zone-center phonon ( $\Gamma$ point) and the zone-boundary phonon ( $\mathrm{K}$ and $\mathrm{K}^{\prime}$ points) are given by

$$
\frac{\hbar}{\tau_{\Gamma}}=\frac{1}{2} \lambda_{\Gamma} \frac{2 \pi \gamma}{L}, \quad \frac{\hbar}{\tau_{K}}=\lambda_{K} \frac{2 \pi \gamma}{L},
$$

where only the backscattering contribution was chosen for the $\Gamma$ phonon and is dominant for the zone-boundary phonon. The corresponding mean free path becomes

$$
\Lambda_{\Gamma}=\frac{\gamma}{\hbar} \tau_{\Gamma}=\frac{L}{\pi \lambda_{\Gamma}}, \quad \Lambda_{K}=\frac{\gamma}{\hbar} \tau_{K}=\frac{L}{2 \pi \lambda_{K}} .
$$

These are written as

$$
\Lambda_{\Gamma}=109 \times\left(\frac{2}{\beta_{\Gamma}}\right)^{2} \times L, \quad \Lambda_{K}=45 \times\left(\frac{2}{\beta_{K}}\right)^{2} \times L .
$$

The results in metallic nanotubes are in reasonable agreement with those calculated using results of LDA calculations of the band structure and coupling constants if we use appropriate parameters for $\beta_{\Gamma} \approx \beta_{K}{ }^{59)}$

\section{$\S 5$. Phonon Self-Energy}

\subsection{Graphene}

With the use of eq. (3.5) for $\boldsymbol{k}^{\prime}=\boldsymbol{k}$, the self-energy of the phonon at the $\mathrm{K}$ point becomes

$$
\Pi^{K}\left(\omega_{K}\right)=-g_{\mathrm{s}} \lambda_{K} \int_{0}^{\infty} \varepsilon \mathrm{d} \varepsilon \frac{-4 \varepsilon[f(-\varepsilon)-f(\varepsilon)]}{\left(\hbar \omega_{K}+\mathrm{i} \Gamma\right)^{2}-4 \varepsilon^{2}},
$$

where $g_{\mathrm{s}}=2$ is the spin degeneracy and $\Gamma$ is a phenomenological broadening parameter due to disorder. By subtracting the contribution for undoped graphene, we redefine the self-energy as

$$
\Pi^{K}\left(\omega_{K}\right)=-g_{\mathrm{s}} \lambda_{K} \int_{0}^{\infty} \varepsilon \mathrm{d} \varepsilon\left(\frac{-4 \varepsilon[f(-\varepsilon)-f(\varepsilon)]}{\left(\hbar \omega_{K}+\mathrm{i} \Gamma\right)^{2}-4 \varepsilon^{2}}-\frac{1}{\varepsilon}\right) .
$$

The corresponding expression for the $\Gamma$ phonon is ${ }^{3)}$

$$
\Pi^{\Gamma}\left(\omega_{\Gamma}\right)=-\frac{1}{2} g_{\mathrm{s}} \lambda_{\Gamma} \int_{0}^{\infty} \varepsilon \mathrm{d} \varepsilon\left(\frac{-4 \varepsilon[f(-\varepsilon)-f(\varepsilon)]}{\left(\hbar \omega_{\Gamma}+\mathrm{i} \Gamma\right)^{2}-4 \varepsilon^{2}}-\frac{1}{\varepsilon}\right) .
$$

This shows that the self-energy of the $\mathrm{K}$ phonon exhibits the same dependence on the electron concentration ${ }^{35)}$ except that both coupling parameter and the frequency are replaced by those of the $\Gamma$ phonons and that the self-energy is multiplied by two.

\subsection{Nanotube}

The self-energy becomes

$$
\begin{array}{r}
\Pi_{j}^{K}\left(\omega_{K}\right)=-g_{\mathrm{s}} \sum_{s, s^{\prime}} \sum_{k, n, n^{\prime}}\left(\frac{2 \beta_{K} \gamma^{2}}{b^{2}}\right)^{2} \frac{\hbar}{2 N M \omega_{K}} \delta_{n^{\prime}, n+j-\nu} \\
\times\left|\boldsymbol{F}_{s^{\prime} n^{\prime}, k}^{K^{\prime} \dagger} \sigma_{y} \boldsymbol{F}_{s n k}^{K}\right|^{2} \frac{f\left(\varepsilon_{s n k}^{K}\right)-f\left(\varepsilon_{s^{\prime} n^{\prime} k}^{K^{\prime}}\right)}{\hbar \omega_{K}-\varepsilon_{s n k}^{K}+\varepsilon_{s^{\prime} n^{\prime} k}^{K^{\prime}}+\mathrm{i} \Gamma} .
\end{array}
$$

For the replacements $n \rightarrow-n^{\prime}$ and $n^{\prime} \rightarrow-n$ and the exchange of $s$ and $s^{\prime}$, the above matrix element remains the same and so does $\delta_{n^{\prime}, n+j-\nu}$. Therefore, after exchanging $n$ and $n^{\prime}$, we have

$$
\Pi_{j}^{K}\left(\omega_{K}\right)=\Pi_{-j}^{K^{\prime}}\left(\omega_{K}\right),
$$

as is expected. In general, the self-energy shift is different from that of longitudinal and transverse phonons at the $\Gamma$ point in nanotubes. ${ }^{2)}$

In metallic nanotubes, we have $\kappa_{-\nu}(n)=\kappa_{\nu}(n)=$ $\kappa(n) \equiv 2 \pi n / L$ and $\varepsilon_{s n k}^{K}=\varepsilon_{s n k}^{K^{\prime}}$ (written as $\varepsilon_{s n k}$ for simplicity). For the mode $j=0$, in particular, the states with $s=s^{\prime}$ do not contribute to the self-energy and therefore we can set $\left|\boldsymbol{F}_{s^{\prime} n^{\prime}, k}^{K^{\prime} \dagger} \sigma_{y} \boldsymbol{F}_{s n k}^{K}\right|^{2}=1$. Then, the self-energy becomes exactly the sum of those of the longitudinal and transverse modes at the $\Gamma$ point except that the coupling parameters and the phonon frequency are replaced by those for the $\mathrm{K}$ phonon. This corresponds to the appearance of a factor two in the self-energy of the $\mathrm{K}$ phonon in comparison with that of the $\Gamma$ phonon in graphene shown in the previous section. In fact, for the 
$\Gamma$ phonons, the self-energy is given by

$$
\begin{aligned}
& \Pi_{ \pm}\left(\omega_{\Gamma}\right)=-g_{\mathrm{v}} g_{\mathrm{s}} \sum_{s, s^{\prime}} \sum_{k, n}\left(\frac{\sqrt{2} \beta_{\Gamma} \gamma^{2}}{b^{2}}\right)^{2} \frac{\hbar}{2 N M \omega_{K}} \\
& \times \frac{1}{2}\left(1 \pm \frac{s s^{\prime}\left[\kappa(n)^{2}-k^{2}\right]}{\kappa(n)^{2}+k^{2}}\right) \frac{f\left(\varepsilon_{s n k}\right)-f\left(\varepsilon_{s^{\prime} n k}\right)}{\hbar \omega_{\Gamma}-\varepsilon_{s n k}+\varepsilon_{s^{\prime} n k}+\mathrm{i} \Gamma}
\end{aligned}
$$

where + and - correspond to the transverse and longitudinal modes, respectively, and $g_{\mathrm{v}}=2$ is the valley degeneracy associated with $\mathrm{K}$ and $\mathrm{K}^{\prime}$ points. ${ }^{2)}$

The self-energy for mode $j \neq 0$ involves interband transitions between $n$ and $n+j$. In this case, the phonon energy $\omega_{K}$ in the denominator can safely be neglected and therefore the self-energy becomes much smaller. The same is applicable to all the modes in semiconducting nanotubes. The self-energy is in general of the same order of magnitude as that of the optical phonons at the $\Gamma$ point in semiconducting nanotubes with similar diameter.

\section{§. Summary}

Zone-boundary phonons at the $\mathrm{K}$ point have been described in a valence-force-field model and then converted into a continuum model. Only a mode with the highest frequency corresponding to a Kekulé-type distortion gives rise to inter-valley electron scattering between the $\mathrm{K}$ and $\mathrm{K}^{\prime}$ points. An effective Hamiltonian for this interaction has been obtained and used for the estimation of the inter-valley scattering in monolayer graphene and carbon nanotubes. It has been shown that inter-valley scattering by zone-boundary phonons is dominant in the high-field transport because of the larger coupling constant and the lower phonon energy than for zone-center phonons.

\section{Acknowledgments}

This work was supported in part by a 21st Century COE Program at Tokyo Tech "Nanometer-Scale Quantum Physics" and by Grants-in-Aid for Scientific Research and for Priority Area "Carbon Nanotube Nano-Electronics" from Ministry of Education, Culture, Sports, Science and Technology Japan.

\section{Appendix A: Interaction Hamiltonian}

In the following we shall derive the interaction Hamiltonian (2.52). Equation (2.3) can be rewritten as

$$
\begin{aligned}
\psi_{A}\left(\boldsymbol{R}_{A}\right) & =\boldsymbol{a}\left(\boldsymbol{R}_{A}\right)^{\dagger} \boldsymbol{F}_{A}\left(\boldsymbol{R}_{A}\right), \\
\psi_{B}\left(\boldsymbol{R}_{B}\right) & =\boldsymbol{b}\left(\boldsymbol{R}_{B}\right)^{\dagger} \boldsymbol{F}_{B}\left(\boldsymbol{R}_{B}\right),
\end{aligned}
$$

with

and

$$
\boldsymbol{F}_{A}=\left(\begin{array}{c}
F_{A}^{K} \\
F_{A}^{K^{\prime}}
\end{array}\right), \quad \boldsymbol{F}_{B}=\left(\begin{array}{c}
F_{B}^{K} \\
F_{B}^{K^{\prime}}
\end{array}\right)
$$

$$
\begin{aligned}
& \boldsymbol{a}\left(\boldsymbol{R}_{A}\right)=\left(\begin{array}{c}
\mathrm{e}^{-\mathrm{i} \boldsymbol{K} \cdot \boldsymbol{R}_{A}} \\
\mathrm{e}^{-\mathrm{i} \eta} \mathrm{e}^{-\mathrm{i} \boldsymbol{K}^{\prime} \cdot \boldsymbol{R}_{A}}
\end{array}\right), \\
& \boldsymbol{b}\left(\boldsymbol{R}_{B}\right)=\left(\begin{array}{c}
-\omega^{-1} \mathrm{e}^{-\mathrm{i} \eta} \mathrm{e}^{-\mathrm{i} \boldsymbol{K} \cdot \boldsymbol{R}_{B}} \\
\mathrm{e}^{-\mathrm{i} \boldsymbol{K}^{\prime} \cdot \boldsymbol{R}_{B}}
\end{array}\right) .
\end{aligned}
$$

First, we introduce a smoothing function $g(\boldsymbol{r})$ which varies smoothly in the range $|r| \sim a$ and decays rapidly for $|r| \gg a$. It should satisfy the conditions:

$$
\sum_{\boldsymbol{R}_{A}} g\left(\boldsymbol{r}-\boldsymbol{R}_{A}\right)=\sum_{\boldsymbol{R}_{B}} g\left(\boldsymbol{r}-\boldsymbol{R}_{B}\right)=1,
$$

giving

$$
\int d \boldsymbol{r} g\left(\boldsymbol{r}-\boldsymbol{R}_{A}\right)=\int d \boldsymbol{r} g\left(\boldsymbol{r}-\boldsymbol{R}_{B}\right)=\Omega_{0},
$$

where $\Omega_{0}$ is the area of a unit cell given by $\Omega_{0}=\sqrt{3} a^{2} / 2$. The function $g(\boldsymbol{r}-\boldsymbol{R})$ can be replaced by a delta function when it is multiplied by a smooth function such as envelopes, i.e.,

$$
g(\boldsymbol{r}-\boldsymbol{R}) \approx \Omega_{0} \delta(\boldsymbol{r}-\boldsymbol{R})
$$

We substitute eq. (A1) into eq. (2.1), multiply $g\left(\boldsymbol{r}^{\prime}-\boldsymbol{R}_{A}\right) \boldsymbol{a}\left(\boldsymbol{R}_{A}\right)$, and then perform summation over $\boldsymbol{R}_{A}$, where $\boldsymbol{r}^{\prime}=\left(x^{\prime}, y^{\prime}\right)$ in the $x^{\prime} y^{\prime}$ coordinates. Then, the equation becomes

$$
\begin{aligned}
\varepsilon \sum_{\boldsymbol{R}_{A}} g\left(\boldsymbol{r}^{\prime}-\boldsymbol{R}_{A}\right) \boldsymbol{a}\left(\boldsymbol{R}_{A}\right) \boldsymbol{a}\left(\boldsymbol{R}_{A}\right)^{\dagger} \boldsymbol{F}_{A}\left(\boldsymbol{r}^{\prime}\right) \\
=-\gamma_{0} \sum_{l} \sum_{\boldsymbol{R}_{A}} g\left(\boldsymbol{r}^{\prime}-\boldsymbol{R}_{A}\right) \boldsymbol{a}\left(\boldsymbol{R}_{A}\right) \boldsymbol{b}\left(\boldsymbol{R}_{A}-\boldsymbol{\tau}_{l}\right)^{\dagger} \\
\times\left[\boldsymbol{F}_{B}\left(\boldsymbol{r}^{\prime}\right)-\left(\boldsymbol{\tau}_{l} \cdot \frac{\partial}{\partial \boldsymbol{r}^{\prime}}\right) \boldsymbol{F}_{B}\left(\boldsymbol{r}^{\prime}\right)+\cdots\right],
\end{aligned}
$$

where $\boldsymbol{F}_{A}\left(\boldsymbol{R}_{A}\right)$ and $\boldsymbol{F}_{B}\left(\boldsymbol{R}_{A}-\boldsymbol{\tau}_{l}\right)$ have been replaced by $\boldsymbol{F}_{A}\left(\boldsymbol{r}^{\prime}\right)$ and $\boldsymbol{F}_{B}\left(\boldsymbol{r}^{\prime}-\boldsymbol{\tau}_{l}\right)$, respectively, because it is multiplied by $g\left(\boldsymbol{r}^{\prime}-\boldsymbol{R}_{A}\right)$. Noting that

$$
\sum_{\boldsymbol{R}_{A}} g\left(\boldsymbol{r}^{\prime}-\boldsymbol{R}_{A}\right) \boldsymbol{a}\left(\boldsymbol{R}_{A}\right) \boldsymbol{a}\left(\boldsymbol{R}_{A}\right)^{\dagger} \approx\left(\begin{array}{ll}
1 & 0 \\
0 & 1
\end{array}\right)
$$

we immediately obtain the left hand side of eq. (A8) as $\varepsilon \boldsymbol{F}_{A}(\boldsymbol{r})$. As for the right hand side, we should note first that

$$
\begin{aligned}
& \sum_{\boldsymbol{R}_{A}} g\left(\boldsymbol{r}^{\prime}-\boldsymbol{R}_{A}\right) \boldsymbol{a}\left(\boldsymbol{R}_{A}\right) \boldsymbol{b}\left(\boldsymbol{R}_{A}-\boldsymbol{\tau}_{l}\right)^{\dagger} \\
& \approx\left(\begin{array}{cc}
-\omega \mathrm{e}^{\mathrm{i} \eta} \mathrm{e}^{-\mathrm{i} \boldsymbol{K} \cdot \boldsymbol{\tau}_{l}} & 0 \\
0 & \mathrm{e}^{-\mathrm{i} \eta} \mathrm{e}^{-\mathrm{i} \boldsymbol{K}^{\prime} \cdot \boldsymbol{\tau}_{l}}
\end{array}\right) .
\end{aligned}
$$

This immediately leads to the conclusion that the first term in the right hand side of eq. (A8) vanishes identically. To calculate the second term we first note that

$$
\begin{aligned}
\sum_{l} \mathrm{e}^{-\mathrm{i} \boldsymbol{K} \cdot \boldsymbol{\tau}_{l}}\left(\begin{array}{ll}
\tau_{l}^{x} & \tau_{l}^{y}
\end{array}\right)=\frac{\sqrt{3}}{2} \omega^{-1} a(+\mathrm{i}+1), \\
\sum_{l} \mathrm{e}^{-\mathrm{i} \boldsymbol{K}^{\prime} \cdot \boldsymbol{\tau}_{l}}\left(\begin{array}{ll}
\tau_{l}^{x} & \tau_{l}^{y}
\end{array}\right)=\frac{\sqrt{3}}{2} a\left(\begin{array}{ll}
-\mathrm{i} & +1
\end{array}\right),
\end{aligned}
$$

and the final result is

$$
\varepsilon \boldsymbol{F}_{A}(\boldsymbol{r})=\gamma\left(\begin{array}{cc}
\hat{k}_{x}-\mathrm{i} \hat{k}_{y} & 0 \\
0 & \hat{k}_{x}+\mathrm{i} \hat{k}_{y}
\end{array}\right) \boldsymbol{F}_{B}(\boldsymbol{r}),
$$

where $\boldsymbol{r}^{\prime}$ has been replaced by $\boldsymbol{r}$ and $\hat{\boldsymbol{k}}^{\prime} \equiv-\mathrm{i} \boldsymbol{\nabla}^{\prime}$ by $\hat{\boldsymbol{k}} \equiv-\mathrm{i} \boldsymbol{\nabla}$. The equation for $\boldsymbol{F}_{B}(\boldsymbol{r})$ can be obtained in a similar manner and the full Schrödinger equation is given by eq. (2.4).

In the presence of bond-length change, the extra term in the hopping integral appearing in eq. (2.45) gives 
rise to a term written as $\mathcal{H}_{\text {int }}^{A B} \boldsymbol{F}_{B}\left(\boldsymbol{r}^{\prime}\right)$ in the right-hand side of eq. (A13), where

$$
\begin{array}{r}
\mathcal{H}_{\mathrm{int}}^{A B}=-\frac{1}{b} \frac{\partial \gamma_{0}}{\partial b} \sum_{l} \sum_{\boldsymbol{R}_{A}} g\left(\boldsymbol{r}^{\prime}-\boldsymbol{R}_{A}\right) \boldsymbol{a}\left(\boldsymbol{R}_{A}\right) \boldsymbol{b}\left(\boldsymbol{R}_{A}-\boldsymbol{\tau}_{l}\right)^{\dagger} \\
\times \boldsymbol{\tau}_{l} \cdot\left[\boldsymbol{u}_{A}\left(\boldsymbol{R}_{A}\right)-\boldsymbol{u}_{B}\left(\boldsymbol{R}_{A}-\boldsymbol{\tau}_{l}\right)\right] . \quad(\mathrm{A} 1
\end{array}
$$

We substitute eq. (2.35) into the above. Because of the slowly-varying nature, we can set $\boldsymbol{u}_{A}^{K}\left(\boldsymbol{R}_{A}\right) \approx \boldsymbol{u}_{A}^{K}\left(\boldsymbol{r}^{\prime}\right)$, $\boldsymbol{u}_{B}^{K}\left(\boldsymbol{R}_{A}-\boldsymbol{\tau}_{l}\right) \approx \boldsymbol{u}_{B}^{K}\left(\boldsymbol{r}^{\prime}\right)$, etc. Then, we have

$$
\mathcal{H}_{\mathrm{int}}^{A B} \approx\left(\begin{array}{cc}
0 & h_{\mathrm{int}}^{A} A B \\
h_{\mathrm{int}}^{A B} & 0
\end{array}\right),
$$

with

$$
\begin{aligned}
h_{\text {int }}^{A B} & =\frac{1}{b} \frac{\partial \gamma_{0}}{\partial b} \omega \mathrm{e}^{\mathrm{i}\left(2 \boldsymbol{K}-\boldsymbol{K}^{\prime}\right) \cdot \boldsymbol{\tau}_{1}} \\
& \times \sum_{l} \boldsymbol{\tau}_{l} \cdot\left[\boldsymbol{u}_{A}^{K}\left(\boldsymbol{r}^{\prime}\right) \mathrm{e}^{-\mathrm{i} \boldsymbol{K} \cdot \boldsymbol{\tau}_{l}}-\boldsymbol{u}_{B}\left(\boldsymbol{r}^{\prime}\right) \mathrm{e}^{-2 \mathrm{i} \boldsymbol{K} \cdot \boldsymbol{\tau}_{l}}\right] \\
h_{\mathrm{int}}^{\prime A B} & =-\frac{1}{b} \frac{\partial \gamma_{0}}{\partial b} \mathrm{e}^{\mathrm{i}\left(2 \boldsymbol{K}^{\prime}-\boldsymbol{K}\right) \cdot \boldsymbol{\tau}_{1}} \\
& \times \sum_{l} \boldsymbol{\tau}_{l} \cdot\left[\boldsymbol{u}_{A}^{K^{\prime}}\left(\boldsymbol{r}^{\prime}\right) \mathrm{e}^{-\mathrm{i} \boldsymbol{K}^{\prime} \cdot \boldsymbol{\tau}_{l}}-\boldsymbol{u}_{B}^{K^{\prime}}\left(\boldsymbol{r}^{\prime}\right) \mathrm{e}^{-2 \mathrm{i} \boldsymbol{K}^{\prime} \cdot \boldsymbol{\tau}_{l}}\right] .
\end{aligned}
$$

We have

$$
\begin{aligned}
& \mathrm{e}^{\mathrm{i}\left(2 \boldsymbol{K}^{\prime}-\boldsymbol{K}\right) \cdot \boldsymbol{\tau}_{1}}=\mathrm{e}^{\mathrm{i}\left(2 \boldsymbol{K}-\boldsymbol{K}^{\prime}\right) \cdot \boldsymbol{\tau}_{1}}=\omega^{-1}, \\
& \sum_{l} \boldsymbol{\tau}_{l} \mathrm{e}^{-2 \mathrm{i} \boldsymbol{K}^{\prime} \cdot \boldsymbol{\tau}_{l}}=\frac{\sqrt{3}}{2} a(+\mathrm{i}, 1), \\
& \sum_{l} \boldsymbol{\tau}_{l} \mathrm{e}^{-2 \mathrm{i} \boldsymbol{K} \cdot \boldsymbol{\tau}_{l}}=\omega \frac{\sqrt{3}}{2} a(-\mathrm{i}, 1) .
\end{aligned}
$$

Then, we have

$$
\begin{aligned}
h_{\mathrm{int}}^{A B} & =\frac{1}{b} \frac{\partial \gamma_{0}}{\partial b} \mathrm{i} \omega \frac{\sqrt{3}}{2} a\left[\omega\left(u_{A x}^{K}-\mathrm{i} u_{A y}^{K}\right)+u_{B x}^{K}+\mathrm{i} u_{B y}^{K}\right] \\
& =\frac{1}{b} \frac{\partial \gamma_{0}}{\partial b} \mathrm{i} \omega \sqrt{3} a \boldsymbol{e}_{K}^{\dagger} \boldsymbol{U}_{K}(\boldsymbol{r}), \\
h_{\mathrm{int}}^{\prime A B} & =\frac{1}{b} \frac{\partial \gamma_{0}}{\partial b} \mathrm{i} \omega^{-1} \frac{\sqrt{3}}{2} a\left[u_{A x}^{K^{\prime}}+\mathrm{i} u_{A y}^{K^{\prime}}+u_{B x}^{K^{\prime}}-\mathrm{i} u_{B y}^{K^{\prime}}\right] \\
& =\frac{1}{b} \frac{\partial \gamma_{0}}{\partial b} \mathrm{i} \omega^{-1} \sqrt{3} a \boldsymbol{e}_{K^{\prime}}^{\dagger} \boldsymbol{U}_{K^{\prime}}(\boldsymbol{r}),
\end{aligned}
$$

where we have replaced $\boldsymbol{r}^{\prime}$ by $\boldsymbol{r}$. The corresponding contribution for $\boldsymbol{F}_{B}(\boldsymbol{r})$ can be calculated similarly, and after some rearrangement we get the Hamiltonian (2.52).

\section{Appendix B: Time Reversal Symmetry}

In general, under a time-reversal operation, an operator $\mathcal{O}$ transforms to

$$
\mathcal{O}^{T}=U^{t} \mathcal{O} U^{-1}
$$

where ${ }^{t} \mathcal{O}$ is the transpose of $\mathcal{O}$ and $U$ represents a unitary operator. ${ }^{68)}$ The time-reversal operation for wavefunctions is given by

$$
\mathcal{T}=U \mathcal{C},
$$

where $\mathcal{C}$ is the operator taking the complex conjugate of the expression following it. When $\mathcal{O}$ is a Hermitian operator, the transformation (B1) becomes

$$
\mathcal{O}^{T}=\mathcal{T} \mathcal{O} \mathcal{T}^{-1}
$$

where $\mathcal{C}^{-1}=\mathcal{C}$.

The Hamiltonian should satisfy $\mathcal{H}^{T}=\mathcal{H}$ in the presence of the symmetry. For the Hamiltonian (2.4), there exist two distinct operations leading to this symmetry. One is the real time-reversal operation

$$
\mathcal{T}=\left(\begin{array}{cc}
0 & \sigma_{z} \\
\sigma_{z} & 0
\end{array}\right) \mathcal{C},
$$

and another is the special time-reversal operation

$$
\mathcal{S}=-\mathrm{i}\left(\begin{array}{cc}
\sigma_{y} & 0 \\
0 & \sigma_{y}
\end{array}\right) \mathcal{C} .
$$

There exists a crucial difference between $\mathcal{T}$ and $\mathcal{S}$ for double operation: $\mathcal{T}^{2}=1$ and $\mathcal{S}^{2}=-1$.

The real time-reversal operation corresponds to $\mathcal{T}$. On the other hand, the special time-reversal operation, $\mathcal{S}$, transforms the electron with the wave vector $\boldsymbol{k}$ to that with $-\boldsymbol{k}$ in the same valley so that the operation is closed within each valley. Therefore, $\mathcal{S}$ essentially works for a time-reversed operation for electron motion when the valley index can be omitted, or there is no inter-valley scattering. The invariance under the operation of $\mathcal{S}$ adds a new symmetry to the system.

The system invariant under $\mathcal{S}$ belongs to the symplectic universality class. The deformation potential (2.59) for acoustic phonons is invariant under $\mathcal{S}$, while the higher-order term (2.62) and the interaction Hamiltonian (2.58) for optical phonons change the symmetry into unitary. On the other hand, the interaction with zone-boundary phonons makes the $\mathcal{S}$ symmetry irrelevant. As a result, the system has the trivial $\mathcal{T}$ symmetry, belonging to the usual orthogonal universality class. This symmetry crossover gives rise to unique transport properties in graphene and carbon nanotubes. ${ }^{9,33,45-50)}$

\section{References}

1) H. Suzuura and T. Ando, Physica E 6 (2000) 864; Mol. Cryst. Liq. Cryst. 340 (2000) 731; AIP Conf. Proc. 590 (2001) 269; Phys. Rev. B 65 (2002) 235412.

2) K. Ishikawa and T. Ando, J. Phys. Soc. Jpn. 75 (2006) 084713.

3) T. Ando, J. Phys. Soc. Jpn. 75 (2006) 124701; J. Phys. Soc. Jpn. 76 (2007) 024712; J. Phys. Soc. Jpn. 76 (2007) 104711.

4) J. W. McClure, Phys. Rev. 104 (1956) 666.

5) T. Ando, J. Phys. Soc. Jpn. 74 (2005) 777.

6) N. H. Shon and T. Ando, J. Phys. Soc. Jpn. 67 (1998) 2421.

7) Y. Zheng and T. Ando, Phys. Rev. B 65 (2002) 245420.

8) T. Ando, Y. Zheng, and H. Suzuura, J. Phys. Soc. Jpn. 71 (2002) 1318.

9) H. Suzuura and T. Ando, Phys. Rev. Lett. 89 (2002) 266603.

10) T. Ando, A. B. Fowler, and F. Stern, Rev. Mod. Phys. 54 (1982) 437 and references cited therein.

11) K. S. Novoselov, A. K. Geim, S. V. Morozov, D. Jiang, Y. Zhang, S. V. Dubonos, I. V. Grigorieva, 
and A. A. Firsov, Science 306 (2004) 666.

12) K. S. Novoselov, A. K. Geim, S. V. Morozov, D. Jiang, M. I. Katsnelson, I. V. Grigorieva, S. V. Dubonos, and A. A. Firsov, Nature 438 (2005) 197.

13) Y. Zhang, Y.-W. Tan, H. L. Stormer, and P. Kim, Nature 438 (2005) 201.

14) M. L. Sadowski, G. Martinez, M. Potemski, C. Berger, and W. A. de Heer, Phys. Rev. Lett. 97 (2006) 266405.

15) S. V. Morozov, K. S. Novoselov, M. I. Katsnelson, F. Schedin, L. A. Ponomarenko, D. Jiang, and A. K. Geim, Phys. Rev. Lett. 97 (2006) 016801.

16) T. Ohta, A. Bostwick, T. Seyller, K. Horn, and E. Rotenberg, Science 313 (2006) 951

17) T. Ohta, A. Bostwick, J. L. McChesney, T. Seyller, K. Horn, and E. Rotenberg, Phys. Rev. Lett. 98 (2007) 206802.

18) J. Jiang, R. Saito, G. G. Samsonidze, A. Jorio, S. G. Chou, G. Dresselhaus, and M. S. Dresselhaus, Phys. Rev. B 75 (2007) 035407.

19) R. S. Deacon, K.-C. Chuang, R. J. Nicholas, K. S. Novoselov, and A. K. Geim, Phys. Rev. B 76 (2007) 081406.

20) J. Martin, N. Akerman, G. Ulbricht, T. Lohmann, J. H. Smet, K. von Klitzing, and A. Yacoby, arXiv: 0705.2180.

21) X.-S. Wu, X.-B. Li, Z.-M. Song, C. Berger, and W. A. de Heer, Phys. Rev. Lett. 98 (2007) 136801.

22) J. Yan, Y. Zhang, P. Kim, and A. Pinczuk, Phys. Rev. Lett. 98 (2007) 166802.

23) S. Pisana, M. Lazzeri, C. Casiraghi, K. S. Novoselov, A. K. Geim, A. C. Ferrari, and F. Mauri, Nat. Mat. 6 (2007) 198.

24) N. Tombros, C. Jozsa, M. Popinciuc, H. T. Jonkman, and B. J. van Wees, Nature 448 (2007) 571.

25) V. P. Gusynin and S. G. Sharapov, Phys. Rev. Lett. 95 (2005) 146801.

26) N. M. R. Peres, F. Guinea, and A. H. Castro Neto, Phys. Rev. B 73 (2006) 125411.

27) H. Kumazaki and D. S. Hirashima, J. Phys. Soc. Jpn. 75 (2006) 053707.

28) K. Nomura and A. H. MacDonald, Phys. Rev. Lett. 96 (2006) 256602.

29) T. Ando, J. Phys. Soc. Jpn. 75 (2006) 074716.

30) I. F. Herbut, Phys. Rev. Lett. 97 (2006) 146401.

31) V. M. Pereira, F. Guinea, J. M. B. Lopes dos Santos, N. M. R. Peres, and A. H. Castro Neto, Phys. Rev. Lett. 96 (2006) 036801.

32) D. V. Khveshchenko, Phys. Rev. Lett. 97 (2006) 036802.

33) E. McCann, K. Kechedzhi, V. I. Falko, H. Suzuura, T. Ando, and B. L. Altshuler, Phys. Rev. Lett. 97 (2006) 146805.

34) M. Lazzeri and F. Mauri, Phys. Rev. B 73 (2006) 165419.

35) A. H. Castro Neto and F. Guinea, Phys. Rev. B 75 (2007) 045404.

36) Ge. G. Samsonidze, E. B. Barros, R. Saito, J. Jiang, G. Dresselhaus, and M. S. Dresselhaus, Phys. Rev. B 75 (2007) 155420.

37) M. Koshino and T. Ando, Phys. Rev. B 75 (2007) 033412 .

38) H. Fukuyama, J. Phys. Soc. Jpn. 76 (2007) 043711.

39) M. Koshino and T. Ando, Phys. Rev. B 75 (2007)
235333

40) M. Koshino and T. Ando, Phys. Rev. B (in press).

41) V. V. Cheianov, V. Falko, and B. L. Altshuler, Science 315 (2007) 1252.

42) T. Ando and T. Nakanishi, J. Phys. Soc. Jpn. 67 (1998) 1704.

43) T. Nakanishi and T. Ando, J. Phys. Soc. Jpn. 68 (1999) 561.

44) T. Ando, T. Nakanishi, and R. Saito, J. Phys. Soc. Jpn. 67 (1998) 2857.

45) T. Ando and H. Suzuura, J. Phys. Soc. Jpn. 71 (2002) 2753.

46) T. Ando, J. Phys. Soc. Jpn. 73 (2004) 1273.

47) T. Ando and K. Akimoto, J. Phys. Soc. Jpn. 73 (2004) 1895.

48) K. Akimoto and T. Ando, J. Phys. Soc. Jpn. 73 (2004) 2194.

49) T. Ando, J. Phys. Soc. Jpn. 75 (2006) 054701.

50) H. Suzuura, Physica E 34 (2006) 674.

51) Z. Yao, C. L. Kane, and C. Dekker, Phys. Rev. Lett. 84 (2000) 2941.

52) A. Javey, J. Guo, M. Paulsson, Q. Wang, D. Mann, M. Lundstrom, and H. Dai, Phys. Rev. Lett. 92 (2004) 106804.

53) J.-Y. Park, S. Rosenblatt, Y. Yaish, V. Sazonova, H. Ustunel, S. Braig, T. A. Arias, P. W. Brouwer, and P. L. McEuen, Nano Lett. 4 (2004) 517.

54) G. D. Mahan, Phys. Rev. B 68 (2003) 125409.

$55)$ G. Pennington and N. Goldsman, Phys. Rev. B 68 (2003) 045426.

56) J. Jiang, R. Saito, A. Gruneis, G. Dresselhaus, and M. S. Dresselhaus, Chem. Phys. Lett. 392 (2004) 383.

57) V. Perebeinos, J. Tersoff, and Ph. Avouris, Phys. Rev. Lett. 94 (2005) 086802.

58) J. Jiang, R. Saito, G. G. Samsonidze, S. G. Chou, A. Jorio, G. Dresselhaus, and M. S. Dresselhaus, Phys. Rev. B 72 (2005) 235408.

59) M. Lazzeri, S. Piscanec, F. Mauri, A. C. Ferrari, and J. Robertson, Phys. Rev. Lett. 95 (2005) 236802.

$60)$ L. M. Woods and G. D. Mahan, Phys. Rev. B 61 (2000) 10651.

61) H. Ajiki, Carbon nanotubes in magnetic fields (Doctor Thesis, University of Tokyo, 1996).

62) N. A. Viet, H. Ajiki, and T. Ando, J. Phys. Soc. Jpn. 63 (1994) 3036.

63) H. Ajiki and T. Ando, J. Phys. Soc. Jpn. 64 (1995) 260.

64) H. Ajiki and T. Ando, J. Phys. Soc. Jpn. 65 (1996) 2976.

65) K. Sugihara, Phys. Rev. B 28 (1983) 2157.

66) T. Ando, J. Phys. Soc. Jpn. 75 (2006) 124701.

67) R. Saito, G. Dresselhaus, and M. S. Dresselhaus, Phys. Rev. B 61 (2000) 2981.

68) M. L. Mehta, Random Matrices (Academic Press, London, 1990).

\section{Figure Captions}

Fig. 1 (a) The lattice structure of a 2D graphite sheet or graphene and various quantities. The $x^{\prime} y^{\prime}$ coordinates are fixed on the the sheet and the $x y$ coordinates are chosen in such a way that the $x$ axis is along chiral vector $\boldsymbol{L}$ in the circumference direction and $y$ axis in the axis direction of a carbon nanotube. $\eta$ is the chiral angle. (b) The first 
Brillouin zone and $\mathrm{K}$ and $\mathrm{K}^{\prime}$ points. (c) The $x y$ coordinates in a nanotube.

Fig. 2 A schematic illustration of phonons at the K' point. The phonon with the highest frequency $\omega_{1}$ corresponds to the Kekulé distortion, those with $\omega_{2}$ are doubly degenerate at the $K^{\prime}$ point and split linearly in proportional to the wave vector measured from the $\mathrm{K}^{\prime}$ point, and that with $\omega_{3}$ vanishes with- out the force constant for the bond-angle change.

Fig. 3 A schematic illustration of scattering of an electron with wave vector $k$ due to emission and absorption of phonon with frequency $\omega$. The wave vectors of the scattered electron are denoted by $\pm k_{ \pm}$. For zone-boundary phonons, the initial and final states belong to a different valley ( $\mathrm{K}$ or $\mathrm{K}$ ' points). 

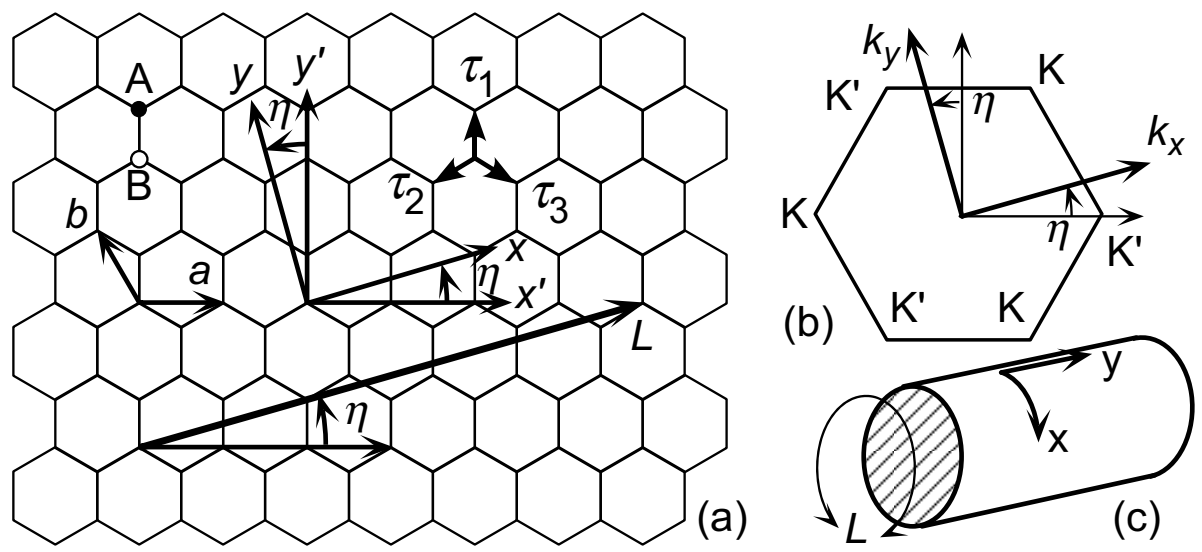

Fig. 1

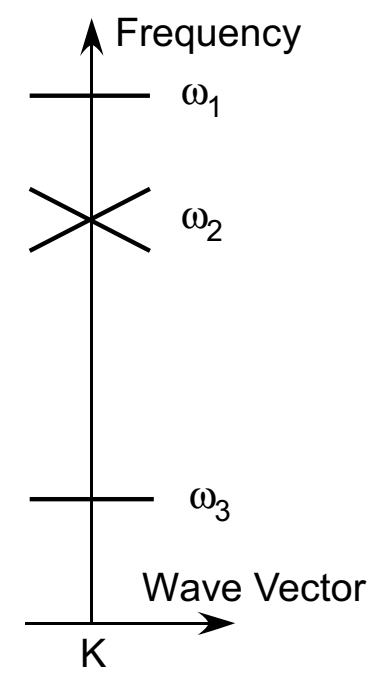

Fig. 2
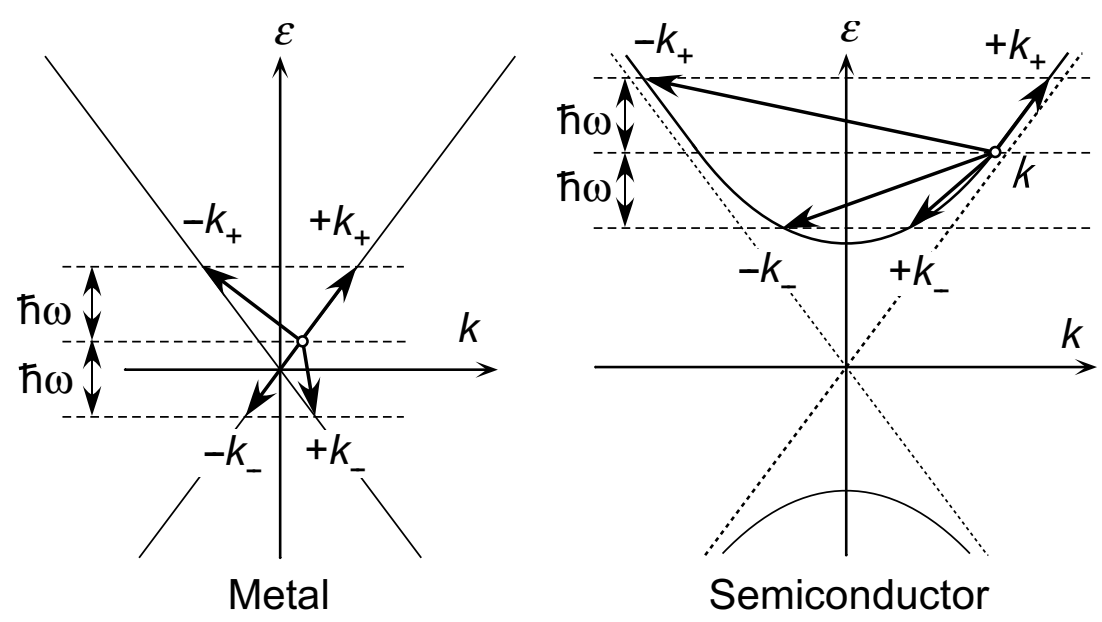

Fig. 3 